\title{
Laparoscopic Imaging Systems
}

It is well known that laparoscopy is the consequence of advances made in the field of medical engineering. Each surgical specialty has different requirement of instruments. Laparoscopy was initially criticized owing to the cost of specialized instruments and possible complications due to these sharp long instruments. Also, it necessitated difficult hand eye coordination. Gradually, the technique gained recognition and respect from the medical fraternity since it drastically reduced many of the complications of the open procedure. Minimal access surgery has developed rapidly only after grand success of laparoscopic cholecystectomy. Computer aided designing of laparoscopic instruments is an important branch of medical engineering. It is now possible to control the access through microprocessor controlled laparoscopic instruments. New procedures and instruments are innovated regularly which makes it important for the surgeon to be familiar with the developments. Laparoscopy is a technologically dependent surgery and it is expected every surgeon should have reasonably good knowledge of these instruments.

\section{LAPAROSCOPIC TROLLEY}

The mobile laparoscopic video cart is equipped with locking brakes and has four anti-static rollers. The trolley has a drawer and three shelves (Fig. 2.1).

The upper shelves have a tilt adjustment and used for supporting the video monitor unit. Included on the trolley is an electrical supply terminal strip, mounted on the rear of the second shelf (from the top). Recently, ceiling mounted trolleys are launched by many companies which are ergonomically better and consume less space in operation theater.

\section{IMAGING SYSTEMS}

- Light source

- Light cable

- Telescope

- Laparoscopic camera

- Laparoscopic video monitor.

The imaging system is a chain of equipments that are link together in place perfectly and functioning well to produce an excellent laparoscopic image. The break in this

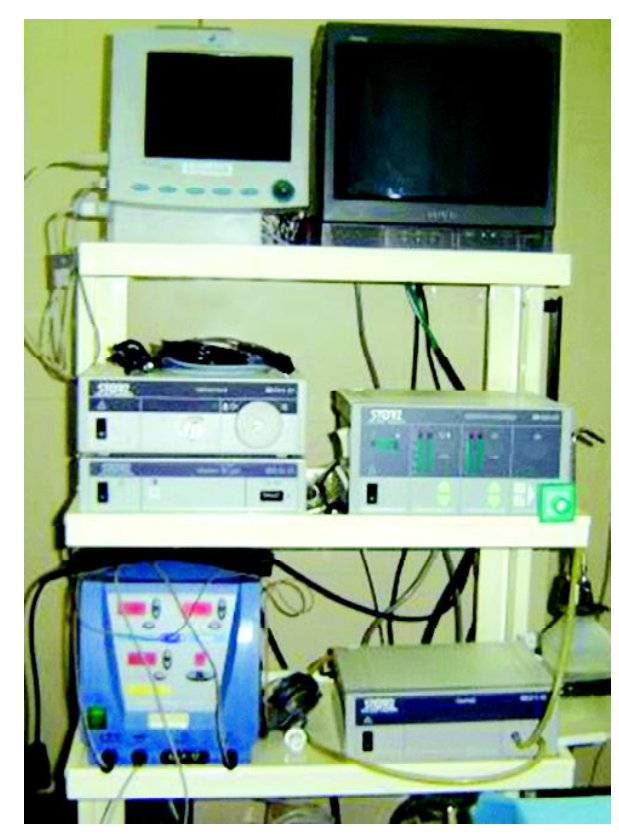

Fig. 2.1: Laparoscopic trolley 
sequential pass of links of the chain will be rendered our imaging system impotent.

The classic imaging chain starts with a light source, and ends in the monitor, requiring seven pieces of equipment, known as the Magnificent Seven: light source, fiber-optic light cable, laparoscope, camera head, video signal processor, video cable, and monitor (Fig. 2.2). This imaging chain is often supported by a cast of VCRs, photo printers, or digital capture devices. The surgeon and the operating room team must work together to ensure optimal equipment function through careful handling of the equipment in the operating room and during the sterilization process. Yet, when the image is poor, many operating teams become paralyzed, unable to function without the aid of a medical engineer. "Understanding can overcome any situation, however, mysterious or insurmountable, it may appear to be." Accordingly, understanding the ( imaging) video system will allow the operating surgeon to do the basic troubleshooting for his or her system and not be totally dependent on nursing or technical staff, especially at night when experienced personnel may not be available. The advent of integrated operating suites has not changed the principles of this basic idea.

\section{THE LIGHT SOURCE}

It is clear and easy to say that, life, recently, is impossible without light, and simply: no light, no laparoscopy. The light source is the often-overlooked soldier of the video laparoscopic system.

High-intensity light is created with bulbs of halogen gas, xenon gas or mercury vapor. The bulbs are available in different wattages" 150 and 300 Watt" and should be chosen based on the type of procedure being performed. Because light is absorbed by blood, any procedure in which bleeding is encountered may require more light. We use the stronger light sources for all advanced laparoscopy. Availability of light is a challenge in many bariatric procedures where the abdominal cavity is large.

A good laparoscopic light source should emit light as much as possible near the natural sun light.

Three types of light source are in use today:

1. Halogen light source

2. Xenon light source

3. Metal halide light source

The output from the light sources is conducted to the telescope by light cables that contain either glass fiber bundles or special fluid.

The halogen light source is used in the medical field since last 20 years, but the spectral temperature of these lights is 3200 Kelvin which makes it too different and too low from natural sunlight. The midday sunlight has approximately 5600 Kelvin color temperature. In practice, the yellow light of the halogen bulb is compensated for in the video camera system by white balancing.

A more suitable light source for laparoscopic cameras involves the creation of an electrical arc in a metal halide system or in xenon. This electrical arc is produced in same way as in flash of photographic camera.

$X$ enon has a more natural color spectrum and a smaller spot size than halogen. The xenon light source emits a spectral temperature of color of approximately 6000 Kelvin on average for a power of $300 \mathrm{~W}$ (Fig. 2.3).

Arc generated lamps have a spectral temperature that gradually decreases with use and white balance is required before each use. The bulb needs replacing after 250 to 500 hours of usage, depending on the type of lamp.

One of the main advantages of the laparoscopy is that of obtaining a virtually microsurgical view compared to that obtained by laparotomy. Quality of the image obtained very much depends on the quantity of light available at each step of optical and electronic system.

The Interface of the laparoscopic team work with a Standard Light Source:

It is essential for the laparoscopic team, particularly the surgeons, to know about all the switch and function of the light source. All essential details of the equipment and all the action required on the part can be found on the operating manual of the product.

Many light sources record and display the hours of service and alert the biomedical medical engineer (or the wellinformed surgeon) when it is time to make a change. When

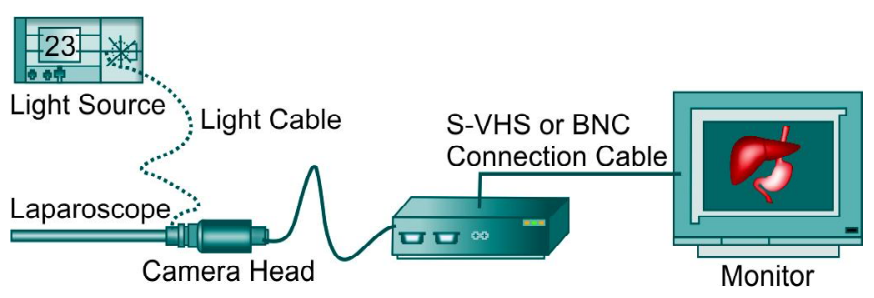

Fig. 2.2: The "Magnificent Seven" of the basic imaging chain

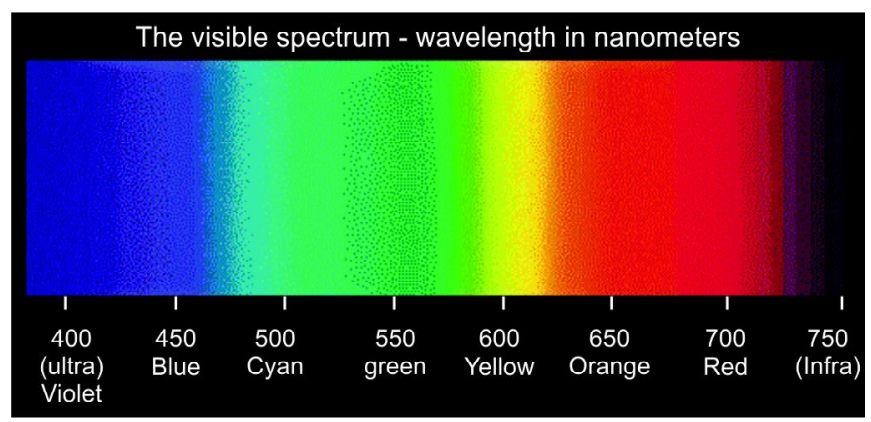

Fig. 2.3: Spectrum of light 
the lifetime rating of the bulb has been exceeded, the subsequent performance of the light source becomes unpredictable, often slowly dwindling until the surgeon just can not produce a well-lit scene despite the fact that a bright light seems to emanate from the laparoscope (Fig. 2.4).

A typical light source consists of:

- A lamp (bulb)

- A heat filter

- A condensing lens

- Manual or automatic intensity control circuit (shutter).

\section{Lamp (Bulb)}

Lamp or bulb is the most important part of the light source.

When the bulb fails, the entire system is out of commission until either the bulb is replaced or a new light is brought to bear. Many light sources record and display the hours of service and alert the biomedical medical engineer (or the well-informed surgeon) when it is time to make a change. When the lifetime rating of the bulb has been exceeded, the subsequent performance of the light source becomes unpredictable, often slowly dwindling until the surgeon just can not produce a well-lit scene despite the fact that a bright light seems to emanate from the laparoscope.

The quality of light depends on the lamp used. Several modern types of light sources are currently available (Fig. 2.5). These light sources mainly differ on the type of bulb used.

Three types of lamp are used more recently:

1. Quartz halogen Incandescent lamp

2. Xenon lamp

3. Metal halide vapor arc lamp.

\section{I-Halogen Bulbs (150-watt) or Tungsten-halogen Bulb}

It is an incandescent lamp with a transparent quartz bulb and a compressed gas filling that includes a halogen. Quartz is used instead of glass to permit higher temperatures, higher currents, and therefore greater light output. The lamp gives brilliant light. The halogen combines with the tungsten evaporated from the hot filament to form a compound that is attracted back to the filament, thus extending the filament's life. The halogen gas is also prevents the evaporated tungsten from condensing on the bulb and darkening it, an effect that reduces the light output of ordinary incandescent lamps.

First used in the late 1960s in motion-picture production, halogen lamps are now also used in automobile headlights, underwater photography, and residential lighting.

Incandescent (to begin to glow): It is so hot to the point of glowing or emitting intense light rays, as an incandescent light bulb.

Quart, one of the commonest of all rock-forming minerals and one of the most important constituents of the

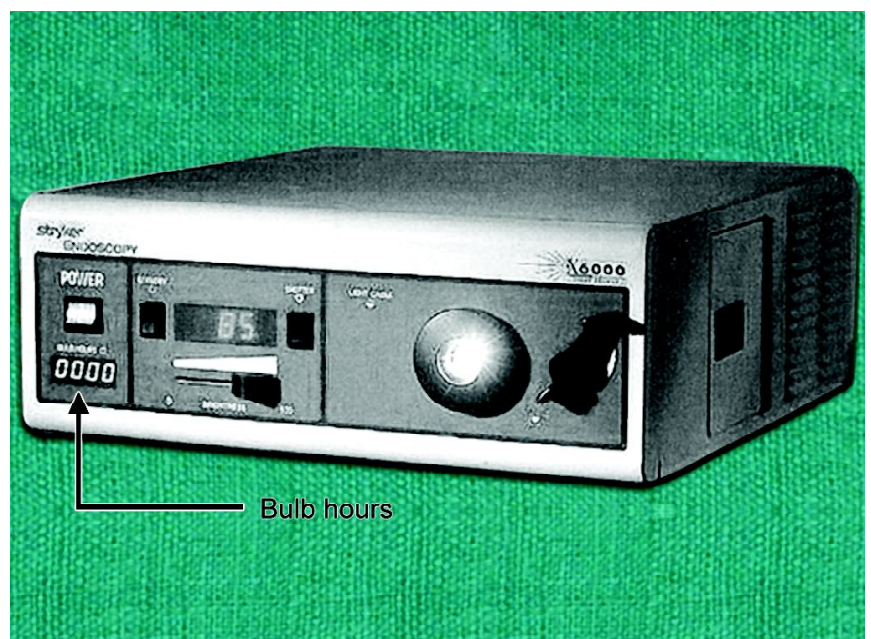

Fig 2.4: Xenon light source: bulb-life display is shown

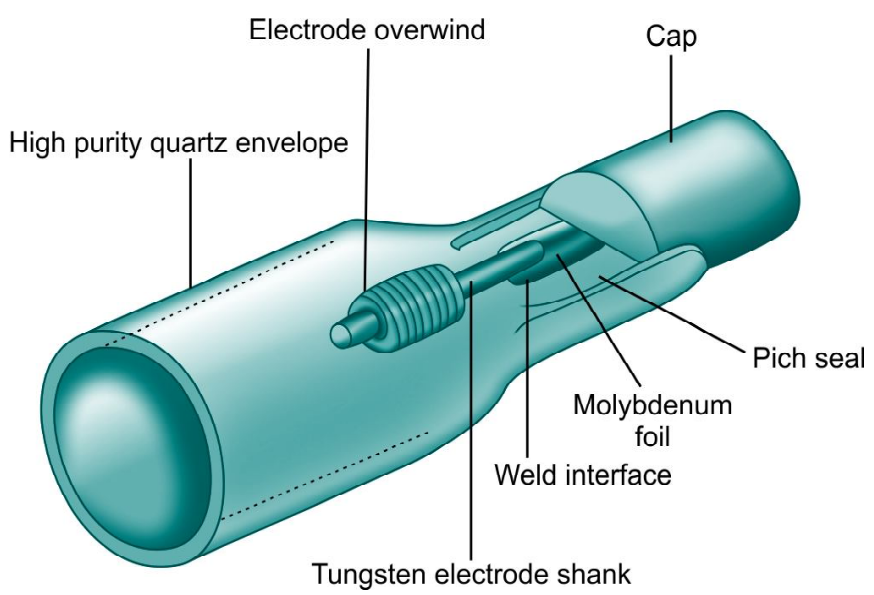

Fig. 2.5: New generation light source bulb

earth's crust. Chemically, it is silicon dioxide, $\mathrm{SiO}_{2}$. It occurs in crystals of the hexagonal system, commonly having the form of a six-sided prism terminating in a six-sided pyramid; the crystals are often distorted and twins are common. Quart may be transparent, translucent, or opaque; it may be colorless or colored.

The halogen lamp takes its name from the halogens included in the gas within its tungsten-filament bulb, added to prolong filament life and increase brightness.

Halogen: Any of the elements of the halogen family, consisting of fluorine, chlorine, bromine, iodine, and astatine. They are all monovalent and readily form negative ions.

$\mathrm{H}$ alogen bulbs provide highly efficient crisp white light source with excellent color rendering. Electrodes in halogen lamps are made of tungsten filament. This is the only metal with a sufficiently high melting temperature and sufficient vapor pressure at elevated temperatures. They use a halogen 
gas that allows bulbs to burn( light) more intensely. Halogen bulbs use low voltages and have an average life of 2,000 hours. The color temperature of halogen lamp is around (5000-5600 K). These lamps are economical and can be used for laparoscopic surgery if low budget setup is required.

\section{II-Xenon Lamps (300-watt)}

Xenon (Symbol Xe): A colorless, odorless, highly unreactive gaseous nonmetallic element found in minute quantities in the atmosphere and extracted commercially from liquefied air. Atomic number is 54 . The radioactive isotope $133 \mathrm{Xe}$, having a half-life of 5.3 days, is used for diagnostic imaging in assessment of pulmonary function, lung imaging, and cerebral blood flow studies.

Xenon lamps consist of a spherical or ellipsoidal envelope made of quart glass, which can withstand high thermal loads and high internal pressure. For ultimate image quality, only the highest-grade clear fused silica quartz is used. It is typically doped, although not visible to the human eye, to absorb harmful UV radiation generated during operation. The color temperature of xenon lamp is about 6000 to 6400 $K$. The operating pressures are tens of atmospheres at times, with surface temperatures exceeding $600^{\circ} \mathrm{C}$.

The smaller, pointed electrode is called the cathode, which supplies the current to the lamp and facilitates the emission of electrons. To supply a sufficient amount of electrons, the cathode material is doped with thorium. The optimum operating temperature of the cathode tip is approximately $2000^{\circ} \mathrm{C}$. To obtain this precise operating temperature, the cathode tip is pointed and in many cases has a groove on the pointed tip to act as a heat choke. This heat choke causes the tip to run at a higher temperature. This configuration of the cathode tip allows for a very high concentration of light from the cathode tip and a very stable arc.

The anode, the larger electrode, receives electrons emitted by the cathode. Once the electrons penetrate the anode face, the resulting energy is converted to heat, most of which radiates away. The large, cylindrical shape of the anode helps to keep the temperature low by radiating the heat from the anode surface.

The advantage of xenon bulb is that, it used a two electrodes (cathode and anode) and there is no filament as in halogen bulb, so it has somewhat a fixed lifetime with an average of 1500 hours.

The two most frequently used types of lamps are halogen and xenon. The main difference between them is in the colors obtained. The xenon lamp has a slightly bluish tint. The light emitted by xenon lamp is more natural compared to halogen lamp. However, most of the cameras at present analyze and compensate these variations by means of automatic "equalization of whites" (2100-10,000 K), which allows the same image to be obtained with both light sources.

A proper white balancing before start of the operation is essential for obtaining a natural color. The white light is composed of equal proportion of red, blue and green color. At the time of white balancing, the camera sets its digital coding for these primary colors to equal proportion, assuming that the target is white. If at the time of white balancing, the telescope is not seeing a perfectly white object, the setup of the camera will be incorrect and the color perception will be poor.

The newer light source of xenon is defined as a cool light but practically it is not completely heat free and it should be cared for ignition hazard.

\section{III-Metal Halide Vapor Arc Lamp (250-watt)}

Halide: $A$ halide is a binary compound, of which one part is a halogen atom and the other part is an element or radical that is less electronegative (or more electropositive) than the halogen, to make afluoride, chloride, bromide, iodide or astatide compound. Many salts are halides. All group 1 metals form halide compounds which are white solids at room temperature.

A halide ion is a halogen atom bearing a negative charge. The halide ions are fluoride $\left(\mathrm{F}^{-}\right)$, chloride $\left(\mathrm{Cl}^{-}\right)$, bromide $\left(\mathrm{Br}^{-}\right)$, iodide $\left(\mathrm{I}^{-}\right)$and astatide $\left(\mathrm{At}^{-}\right)$. Such ions are present in all ionic halide salts.

Metal halides are used in high-intensity discharge lamps called metal halide lamps, such as those used in modern street lights. These are more energy-efficient than mercuryvapor lamps, and have much better color rendition than orange high-pressure sodium lamps. Metal halide lamps are also commonly used in greenhouses or in rainy climates to supplement natural sunlight.

Examples of halide compounds are: sodium chloride $(\mathrm{NaCl})$, potassium chloride $(\mathrm{KCl})$, potassium iodide $(\mathrm{KI})$, lithium chloride ( $\mathrm{LiCl})$, copper (II) chloride $\left(\mathrm{CuCl}_{2}\right)$, silver chloride $(\mathrm{AgCl})$, and chlorine fluoride (CIF).

Metal halide lamps, a member of the high-intensity discharge (HID) family of lamps, produce high light output for their size, making them a compact, powerful, and efficient light source. By adding rare earth metal salts to the mercury vapor lamp, improved luminous efficacy and light color is obtained. Originally created in the late 1960s for industrial use, metal halide lamps are now available in numerous sizes and configurations for commercial and residential applications (Figs 2.6 to 2.8).

Like most HID lamps, metal halide lamps operate under high pressure and temperature, and require special fixtures to operate safely. 


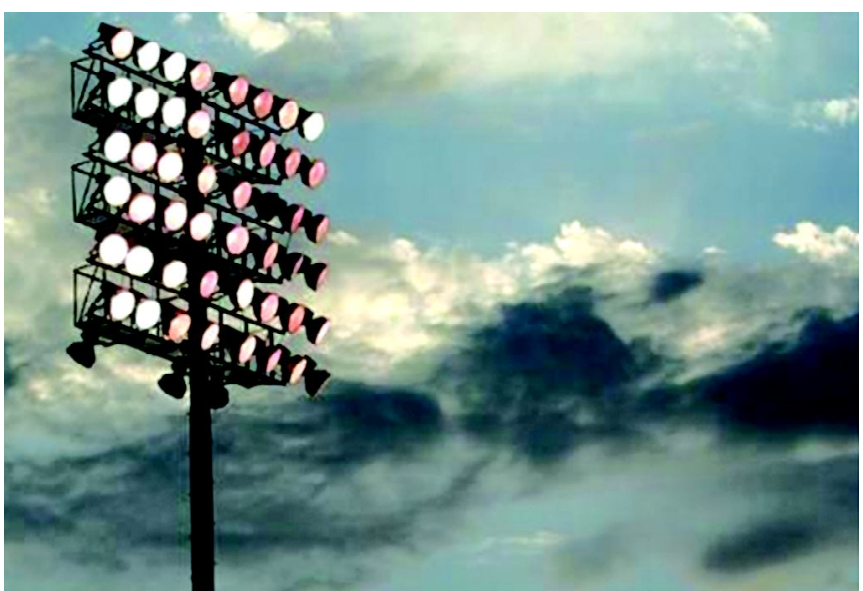

Fig. 2.6: A metal halide gas discharge lighting system provides illumination for a college baseball game. Note the various colors of the lights as they warm up

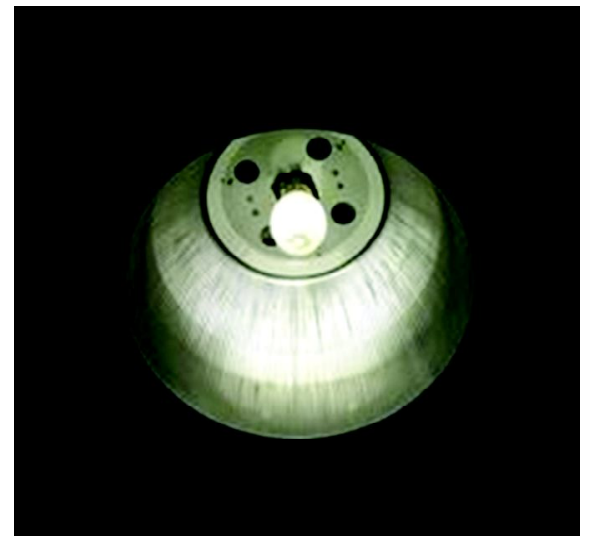

Fig. 2.7: A low-bay light fixture using a high wattage metal halide lamp, of the type used in factories and warehouses

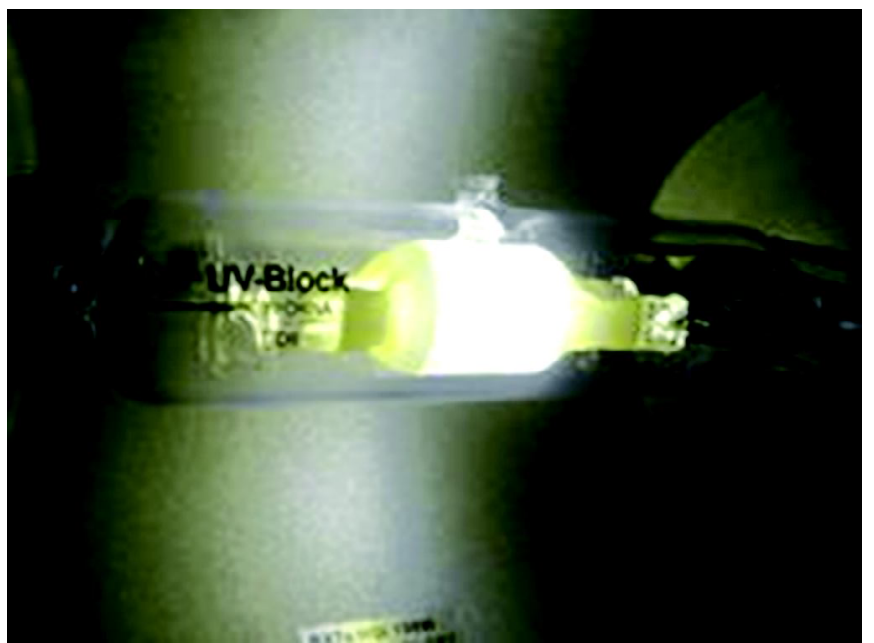

Fig. 2.8: A linear/tubular metal halide lamp lit up at half power
In metal halide lamp, a mixture of compounds (comprising mostly salts of rare earths and halides as well as the mercury which provides the conduction path) is carefully chosen to produce an output which approximates to 'white' light as perceived by the human eye (Fig. 2.9).

There are two type of metal halide lamp generally used. They are iron iodide lamp and gallium iodide lamp. Iron iodide is a broad emitter and enhances the spectral output of the lamp in the $380 \mathrm{~nm}$. G allium iodide has the effect of introducing spectral lines at $403 \mathrm{~nm}$ and $417 \mathrm{~nm}$ of the electromagnetic spectrum (Fig. 2.10).

The intensity of the light delivered by any lamp also depends on the power supply of the source. However, increasing the power poses a real problem as it generates more heat. At present, the improvements made to the cameras means that it is possible to return to reasonable

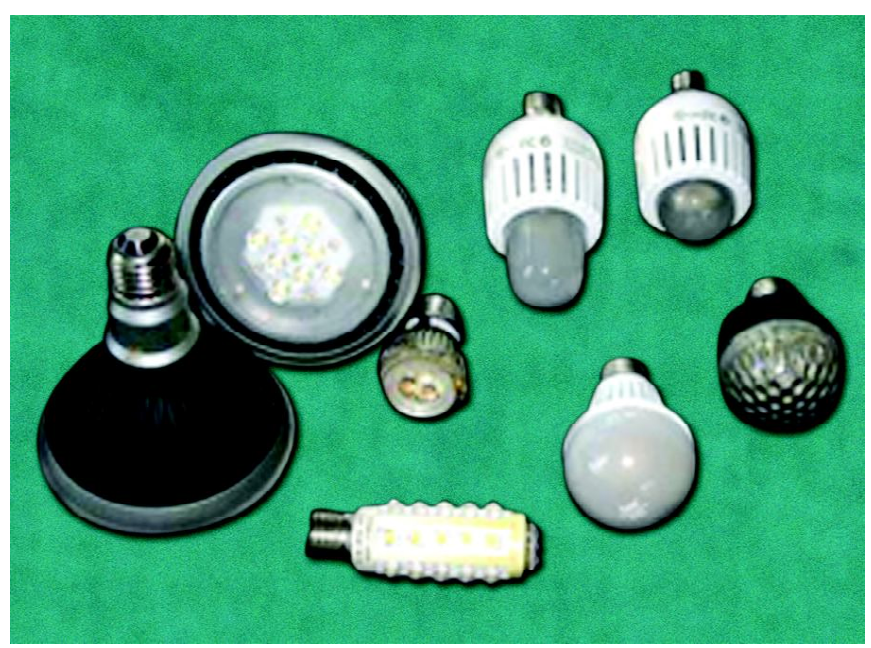

Fig. 2.9: Metal halide bulb

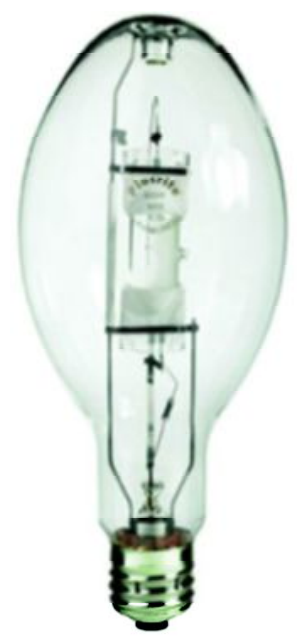

Fig. 2.10: Internal structure of metal halide tube 
power levels of $250 \mathrm{~W}$. H owever, $400 \mathrm{~W}$ units are preferable in order to obtain sufficient illumination of the abdomen even when bleeding causes strong light absorption. It is important to remember that a three chip camera require more light than single chip camera so a $400 \mathrm{~W}$ light source is recommended for 3 chips camera.

\section{Metal Halide Lamp}

\section{Heat Filter}

For 100 percent of energy consumed, a normal light source (a light bulb) converts approximately two percent to light and 98 percent as heat. This heat is mainly due to the infrared spectrum of light and due to obstruction in the pathway of light. If infrared travels through the light cable, the cable will become hot. A heat filter is introduced to filter this infrared in fiberoptic cable. A cool light source lowers this ratio by creating more light, but does not reduce the heat produced to zero. This implies a significant dissipation of heat, which increases as the power rating increases. A cold light is light emitted at low temperatures from a source that is not incandescent, such as fluorescence or phosphorescence. Incandescence is the emission of light (visible electromagnetic radiation) from a hot body as a result of its temperature.

The sources are protected against transmitting too much heat at present. The heat is essentially dissipated in transport, along the cable, in the connection with the endoscope and along the endoscope.

While it is remarkable how little heat is delivered to the tip of the laparoscope, the effects are cumulative. A lighted laparoscope or fiber-optic bundle in direct contact with paper drapes or the patient's skin will cause a burn after 20 or 30 seconds and must be avoided.

Some accidents have been reported due to burning caused by the heat of the optics system. It is therefore important to test the equipment, particularly if assemblies of different brands are used.

\section{Condensing Lens}

The purpose of condensing lens is to converge the light emitted by lamp to the area of light cable input. In most of the light source it is used for increasing the light intensity per square $\mathrm{cm}$ of area.

\section{Manual or Automatic Intensity Control Circuit (Shutter)}

Manual adjustment allows the light source to be adjusted to a power level defined by the surgeon. In video cameras, close-up viewing is hampered in too much light, whereas more distant view is to o dark. To address this, the luminosity of most of the current light sources is adjustable.
The advanced light source system is based on the automatic intensity adjustmenttechnology. The video camera transforms the signal into an electronic signal. This electronic signal is coded in order to be transported. The coding disso ciates the luminance and chrominance of the image. The luminance is the quantity of light of the signal (black and white) that dictates the quality of the final image. When there is to much light for the image (when the endoscope isnear to the tissue), the luminance signal of the oscilloscope increases. On the other hand, when the luminosity is low (distant view or red surroundings), the luminance is low and the electronic signal is much weaker. A good quality luminance signal is calibrated to one millivolt. Overexposed images make the electronic signal pass above one millivolt, whereas underexposed images make the signal drop below one millivolt. Light sources equipped with adjustment analyze the luminance. If the signal is significantly higher than one millivolt, they lower the power and bring the signal back within the standards. Conversely, if the signal is too weak, they increase their intensity.

These systems are extremely valuable, permitting work to be performed at different distances from the target in good viewing conditions. However, the cameras currently available are often equipped with a regulation system, which is capable of automatic gain control in poor light condition and the purchase of a light source with adjustment associated with a camera equipped with an adjustment system, is a double purchase that is unnecessary.

Troubleshooting of Laparoscopic Light Source:

Troubleshooting for inadequate lighting is shown in Table 2.1.

A laparoscopic surgeon should be technically wellacknowledged of the principle of the instrument they are using. The purchase of a costly instrument is not an answer for achieving a good task, ability to handle them is equally important.

\section{Light Cable}

Minimal access surgery depends on the artificial light available in closed body cavity, and before the discovery of

Table 2.1: Troubleshooting of light source

\begin{tabular}{ll}
\hline \multicolumn{1}{c}{ Probable cause } & \multicolumn{1}{c}{ Remedy } \\
\hline $\begin{array}{l}\text { Loose connection at source } \\
\text { or scope }\end{array}$ & Adjust connector \\
Light is on "manual-minimum" & Go to "automatic" \\
Bulb is burned out & Replace bulb \\
Fiberoptics are damaged & Replace light cable \\
Automatic iris adjusting to bright & Dim room lights \\
Reflection from instrument & Re-position instruments, \\
& or switch to "manual" \\
\hline
\end{tabular}


light source and light cable; mirrors were used to reflect the light onto the subject where direct light access was not possible.

In 1954, a major breakthrough in technology occurred in the development of fiberoptic cables (Fig. 2.11). The principle of fiberoptic cable was based on the total internal reflection of light. Light can be conducted along a curved glass rod due to multiple total internal reflections. Light would enter at one end of the fiber and emerge at the other end after numerous internal reflections with virtually all of its intensity.

\section{Total Internal Reflection}

An effect that combines both refraction and reflection is total internal reflection (Fig. 2.12). Consider light coming from a dense medium like water into a less dense medium like air.

When the light coming from the water strikes the surface, part will be reflected and part will be refracted. Measured with respect to the normal line perpendicular to the surface, the reflected light comes off at an angle equal to that at which it entered at, while that for the refracted light is larger than the incident angle. In fact the greater the incident angle, the more the refracted light bends away from the normal. Thus, increasing the angle of incidence from path " 1 " to "2" will eventually reach a point where the refracted angle is $90^{\circ}$, at which point the light appears to emerge along the surface between the water and air. If the angle of incidence is increased further, the refracted light cannot leave the water. It gets completely reflected. The interesting thing about total internal reflection is that it really is total. That is 100 percent of the light gets reflected back into the more dense medium, as long as the angle at which it is incident to the surface is large enough.

Fiberoptics uses this property of light to keep light beams focussed without significant loss (Fig. 2.13).

The light enters the glass cable, and as long as the bending is not too sudden, will be totally internally reflected when it hits the sides, and thus is guided along the cable. This is used in telephone and TV cables to carry the signals. Light as an information carrier is much faster and more efficient than electrons in an electric current. Also, since light rays do not interact with each other (whereas electrons interact via their electric charge), it is possible to pack a large number of different light signals into the same fiberoptics cable without distortion. You are probably most familiar with fiber optics cables in novelty items consisting of thin, multi-colored strands of glass which carry light beams.

Nowadays, there are two types of light cable available:

1. Fiberoptic cable

2. Liquid crystal gel cable.

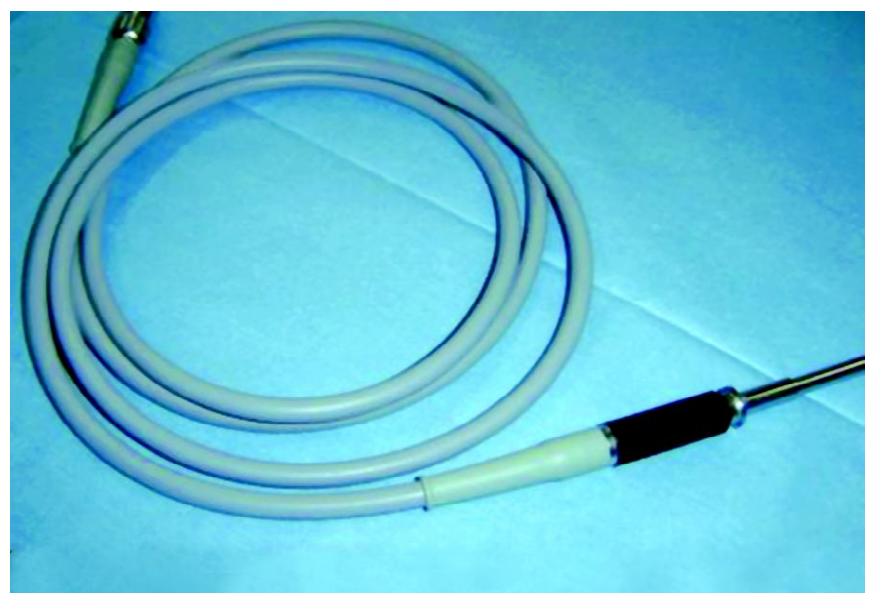

Fig. 2.11: Fiberoptic light cable

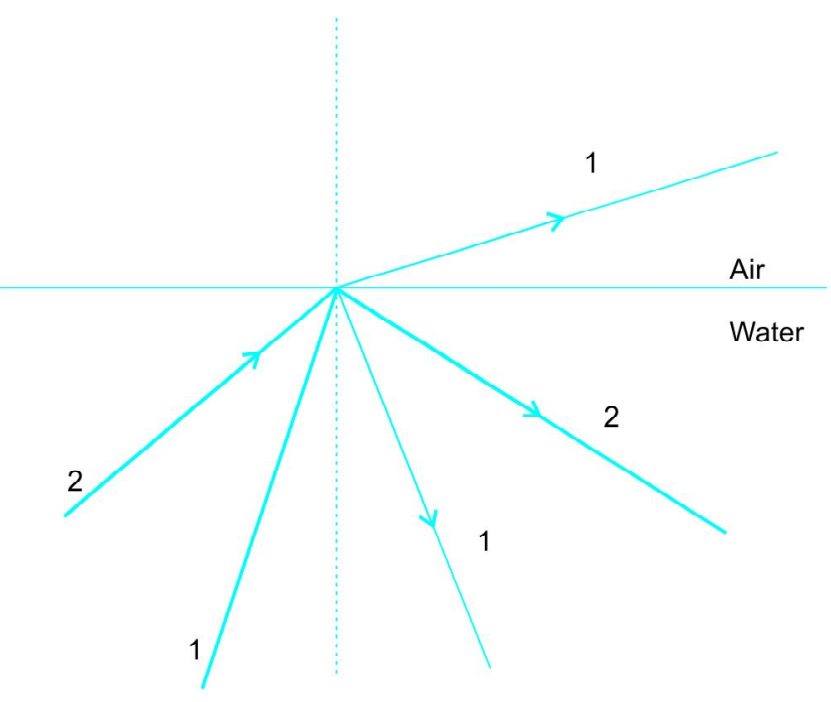

Fig. 2.12: Refraction of light from water into air

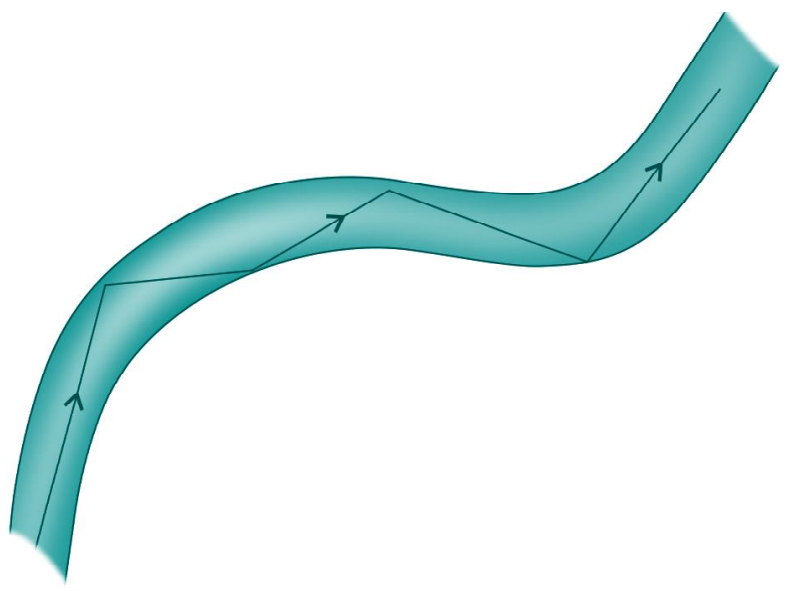

Fig. 2.13: Fiberoptic cable, total internal reflection 


\section{Fiberoptic Cable}

Fiberoptics is the science or technology of light transmission through (a bundle of optical fibers) very fine, flexible glass or plastic fibers.

Fiberoptic cables are made up of a bundle of optical fiber glass thread swaged at both ends. The fiber size used is usually
20 to 150 micron in diameter. A good fiberoptic cable will transmit all the spectrum of light without loss (Fig. 2.14). They have a very high quality of optical transmission, but are fragile.

The light inside these fibers travels on the principle of total internal reflection without losing much of its intensity. The multimode fiber maintains the intensity of light and the light can be passed in a curved path of light cable (Fig. 2.15).

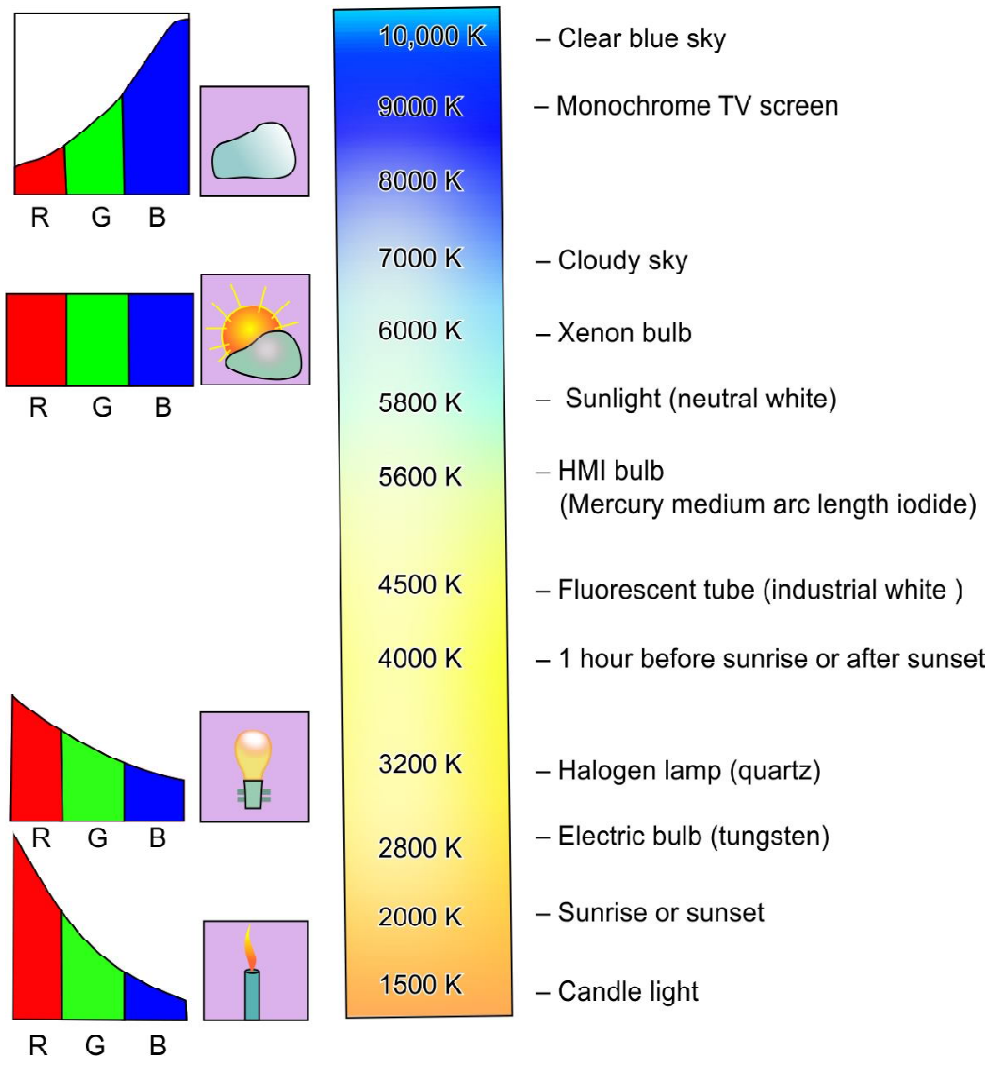

Fig. 2.14: Internal structure of fiberoptic cable

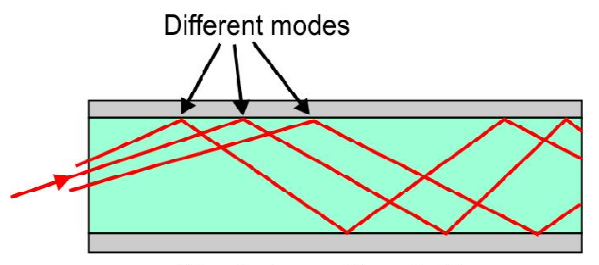

Step-index multimode fiber

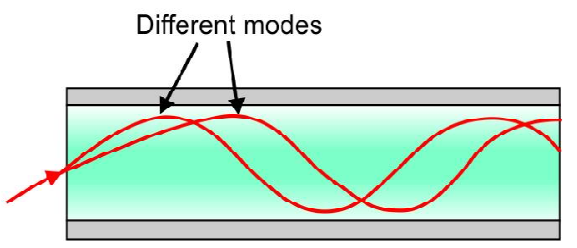

Graded-index multimode fiber

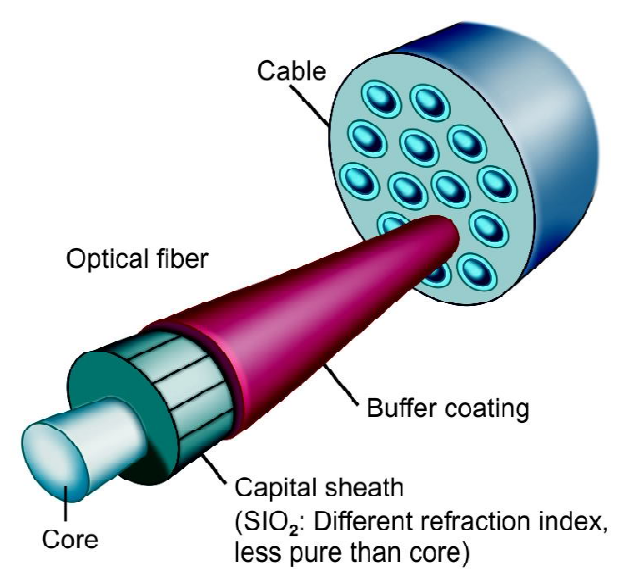

Fig. 2.15: Multimode fiber 
As the light cables are used progressively, some optical fibers break (Fig. 2.16). The loss of optical fibers may be seen when one end of the cable is viewed in daylight. The broken fibers are seen as black spots. To avoid the breakage of these fibers, the curvature radius of light cable should be respected and in any circumstances it should not be less than $15 \mathrm{~cm}$ in radius.

If the heat filter or cooling system of light source does not work properly, the fibers of these light cable is burnt (melt) and it will decrease the intensity of light dramatically (Fig. 2.17). If poor quality fibers are used, it might burn just within a few months of use.

\section{Liquid Crystal Gel Cable}

These cables are made up of a sheath that is filled with a clear optical gel (Liquid crystal).

Crystal (a clear, transparent mineral or glass resembling ice) is a piece of solid substance, such as quart, with a regular shape in which plane faces intersect at definite angles, due to the regular internal structure of its atoms, ions, or molecules. Within a crystal, many identical paralleled-piped unit cells, each containing a group of atoms, are packed together to fill all space (see illustration). In scientific nomenclature, the term crystal is usually short for single crystal, a single periodic arrangement of atoms. Most gems are single crystals. However, many materials are polycrystalline, consisting of many small grains, each of which is a single crystal. For example, most metals are polycrystalline (Fig. 2.18).

\section{Liquid Crystal}

Substance that flows like a liquid but maintains some of the ordered structure characteristic of a crystal. Some organic substances do not melt directly when heated but instead turn from a crystalline solid to a liquid crystalline state. When heated further, a true liquid is formed. Liquid crystals have unique properties. The structures are easily affected by changes in mechanical stress, electromagnetic fields, temperature, and chemical environment.

Liquid Crystal Gel Cables are capable of transmitting up to 30 percent more light than optic fibers. Due to lighter and better color temperature transmission, this cable is recommended in those circumstances, where documentation (movie, photography or TV) is performed.

The quartz swaging at the ends is extremely fragile, especially when the cable is hot. The slightest shock, on a bench for example, can cause the quartz end to crack and thus cause a loss in the transmission of the light.

Gel cables transmit more heat than optical fiber cables. These cables are made more rigid by a metal sheath, which makes them more difficult to maintain and to store. In conclusion, even though the choice is a difficult one, we

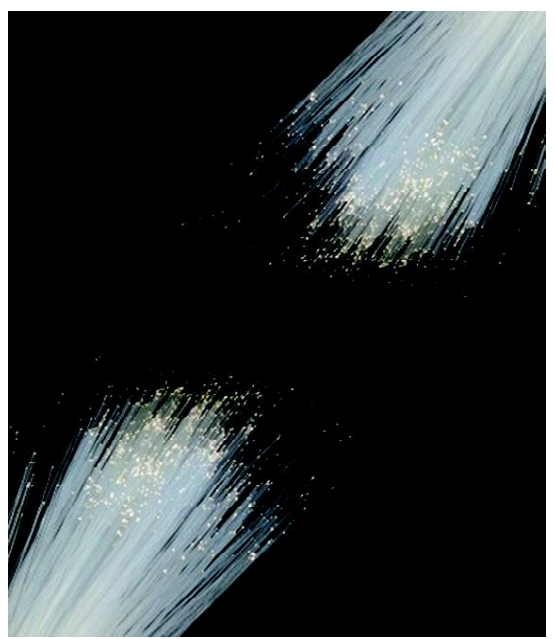

Fig. 2.16: Broken fibers showing significant loss of light
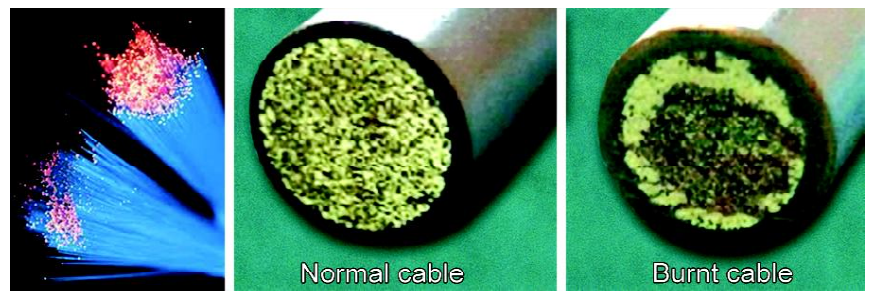

Fig. 2.17: Burnt fiber causes significant reduction in intensity of light

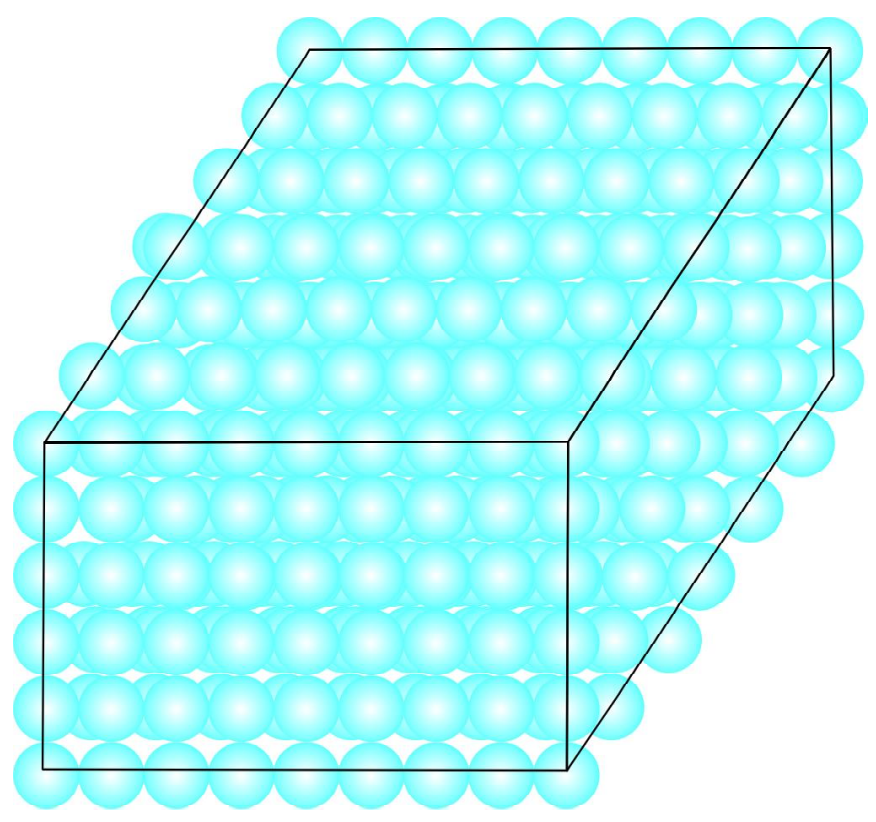

Fig. 2.18: Structure of a simple crystal 
use optical fiber cables, which are as fragile as the gel cables but their flexibility makes them much easier to maintain.

\section{Attachment of the Light Cable to the Light Source}

Conventional attachment has at right angle connection for light source and camera. Recently, new attachment for light cable is available known as DCl interface (Display control interface) (Fig. 2.19). The benefit of this is that it maintains upright orientation regardless of angle of viewing, using autorotation system. It also provides single handed control of the entire endoscope camera system.

\section{Maintenance of Light Cable}

The following points should be followed for the maintenance of light cable:

- Handle them carefully.

- Avoid twisting them.

- After the operation has been completed, the cable should preferably be first disconnected from the endoscope and then disconnected from the light source. In fact, most of the sources currently available have a plug for holding the cable until it cools down.

- The end of the crystal of cable should be periodically cleaned with a cotton swab moistened with alcohol.

- The outer plastic covering of the cable should be cleaned with a mild cleaning agent or disinfectant.

- Distal end of fibero ptic cable should never be placed on or under drapes, or next to the patient, when connected to an illuminated light source. The heat generated from the intensity of light may cause burns to the patient or ignite the drapes (Fig. 2.20).

- The intensity of light source is so high that there is chance of retinal damage if the light will fall directly on eye. Never try to look directly on light source when it is lighted.

\section{Telescope}

There are two type of telescope, rigid and flexible.

The rigid laparoscopic and thoracoscopic telescopes come in a variety of shapes and sizes, and offering several different angles of view. The standard laparoscope consists of a metal shaft between 24 to $33 \mathrm{~cm}$ in length.

There are three important structural differences in telescope available in the market:

- No. of the rod lens: From 6 to 18 rod lens system telescopes

- The Angle of view: B etween 0 degree to $120^{\circ}$ telescopes

- The Diameter: 1.5 to $15 \mathrm{~mm}$ of telescopes.

\section{The Angle of View}

Telescopes offer either a straight-on view with the 0 degree or can be angled at 25 to 30 or 45 to 50 degrees. The 30 -degree telescope provides a total field of view of 152 degrees compared with the 0 -degree telescope, which only provides a field of view of 76 degrees (Fig. 2.21). The $30^{\circ}$ forward oblique angle permits far greater latitude for viewing
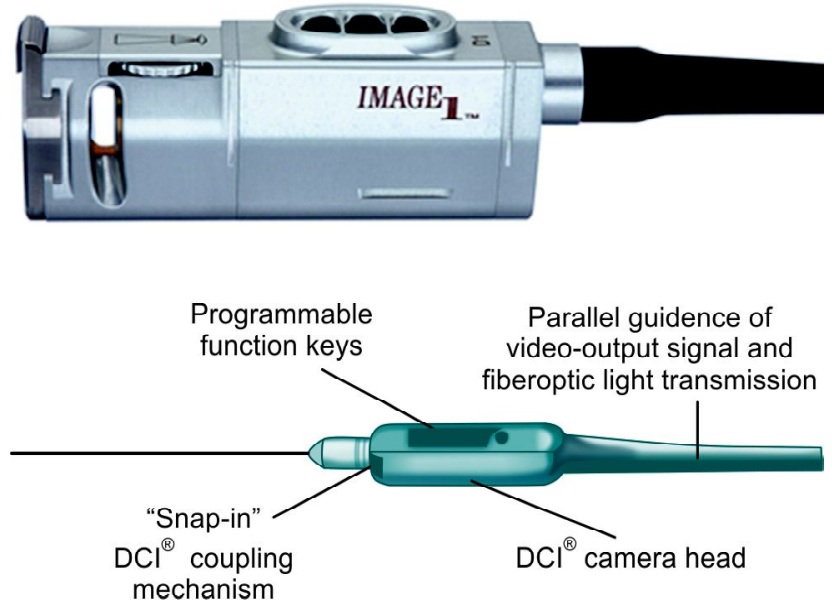

Fig. 2.19: $\mathrm{DCl}$ attachment of light cable

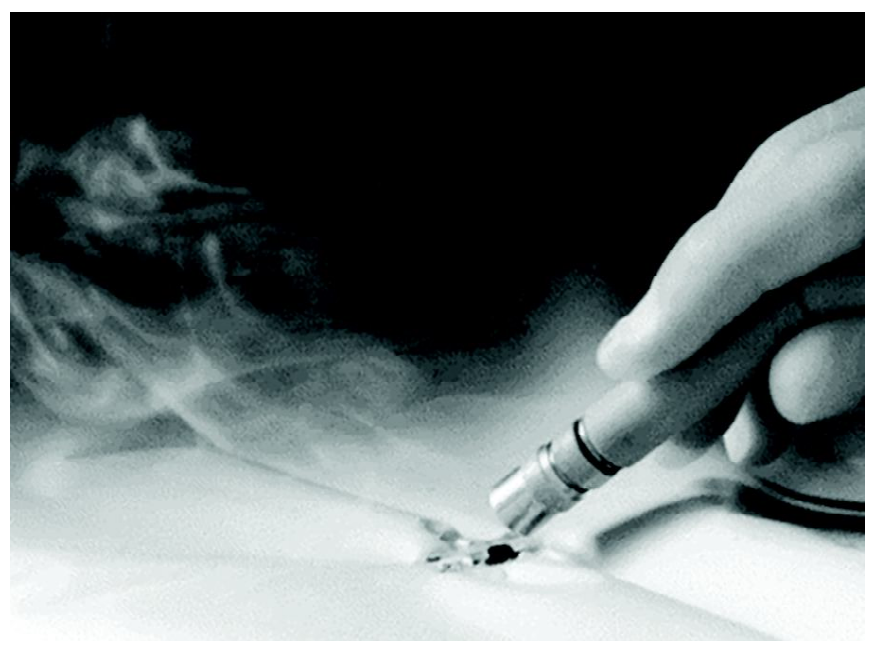

Fig. 2.20: Negligence with light cable can result in burn
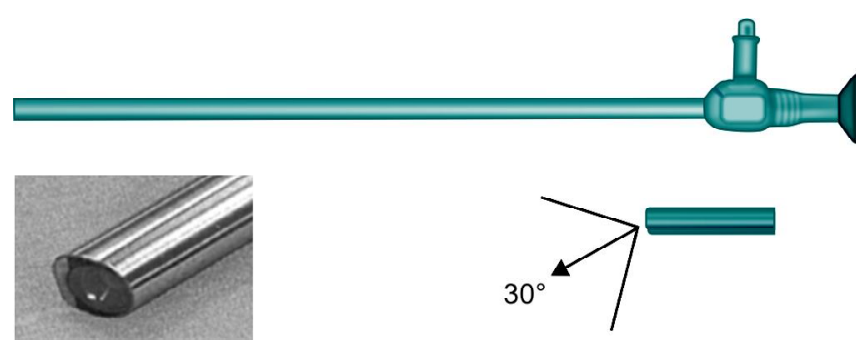

Fig. 2.21: The 30-degree telescope provides a total field of view of 152 degrees compared with the 0 -degree telescope, which provides a field of view of only 76 degrees 
underlying areas under difficult anatomical conditions (Fig. 2.22).

\section{The Diameter}

The most commonly used telescope has a diameter of $10 \mathrm{~mm}$ and provides the greatest light and visual acuity. The next most commonly used telescope is the $5 \mathrm{~mm}$ laparoscope, which can be placed through one of the working ports for an alternative view. Smaller-diameter laparoscopes, down to a $1.1 \mathrm{~mm}$ scope, are available and are used mostly in children. They are not used commonly in adult patients because of an inability to direct enough light into the larger abdominal cavity. Laparoscopes as small as $1 \mathrm{~mm}$ have been produced for diagnostic use. The field of view and picture brightness are dramatic improvements over early designs. "Mini" or "Micro" $2 \mathrm{~mm}$ laparoscopy is reported for diagnostic and even advanced procedures.

One of the problems with working with these smaller laparoscopes (particularly those less than $3.4 \mathrm{~mm}$ ) is that they tend to bend easily, leading to potential damage during surgery.

Full screen $5 \mathrm{~mm}$ laparoscopes with images comparable to many $10 \mathrm{~mm}$ systems are now available.

\section{The Lens System}

There are two lens system designs used with the laparoscopy. The conventional thin lens system and the Hopkins rodlens system design. The thin lens system, which uses a series of objective lenses to transport the image down the laparoscope, is used less commonly. The Hopkins lens system containing a series of quartz-rod lenses that carry

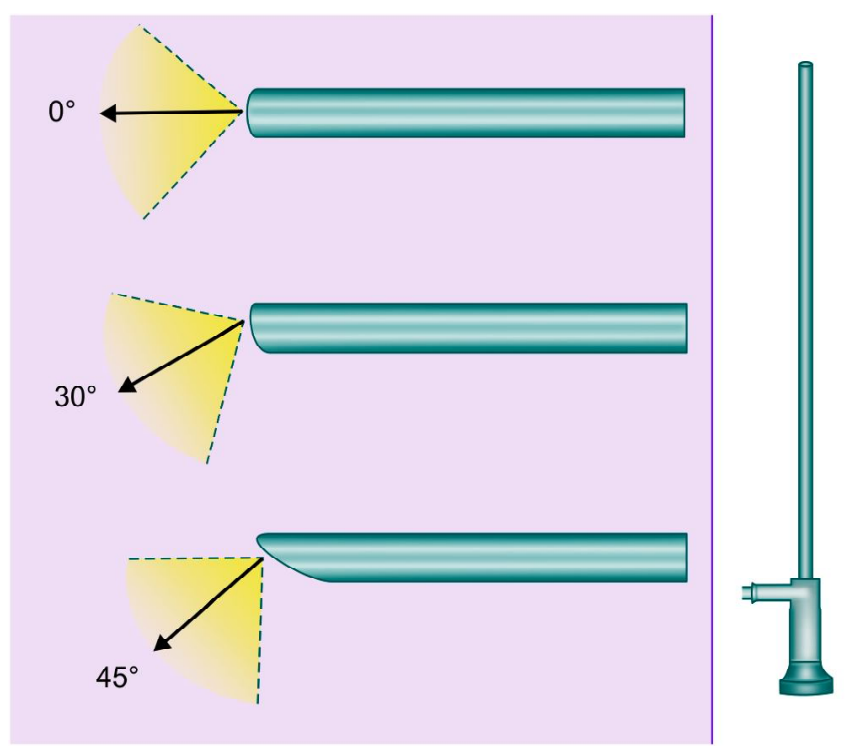

Fig. 2.22: Angle of laparoscope the image through the length of the scope to the eyepiece. Rigid rod lens system provides good resolution and better depth perception (Fig. 2.23). The H opkins lens system uses more glass than air so it has improved light transmission (Fig. 2.24). N ormally used telescope is the Hopkins Forward Oblique Telescope $\left(30^{\circ}\right)$. Its diameter is $10 \mathrm{~mm}$ length $33 \mathrm{~cm}$ and is autoclavable.

At the distal end, it is a front lens complex (inverting real-image lens system, IRILS) which creates an inverted

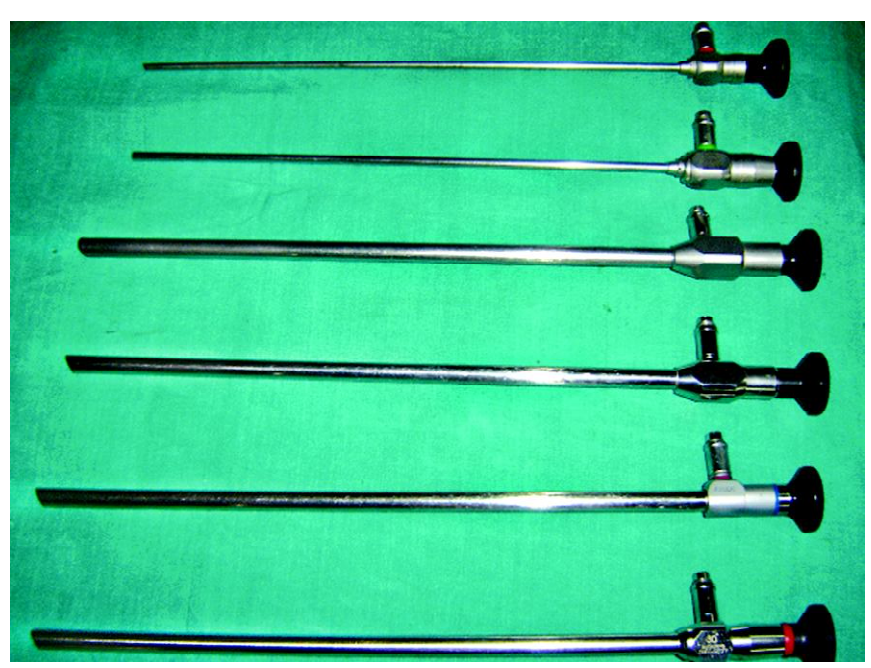

Fig. 2.23: Different types of laparoscope
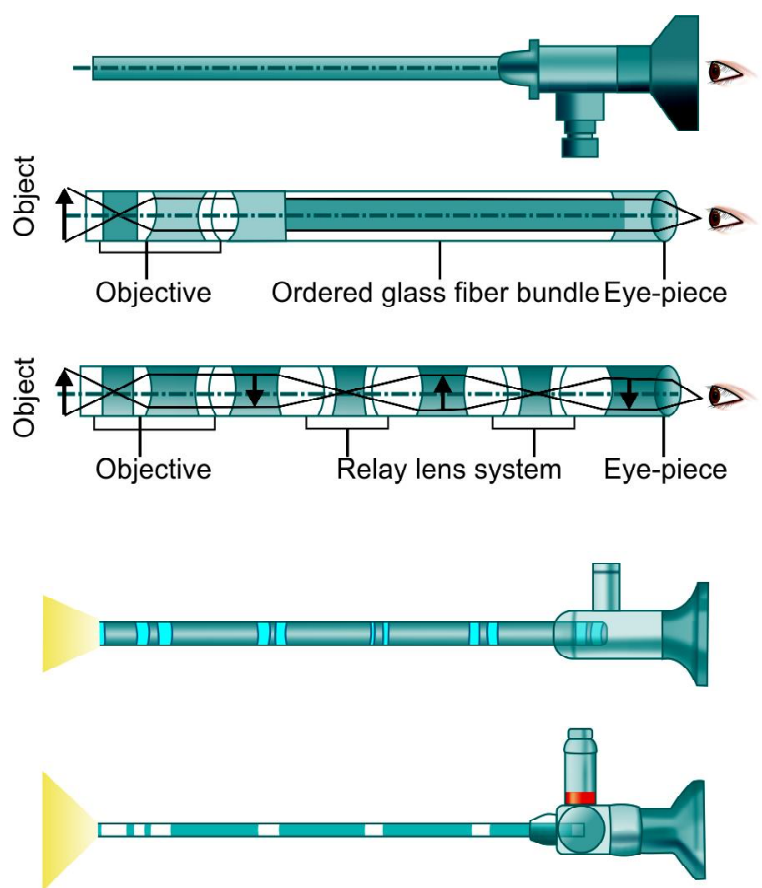

Fig. 2.24: Inside view of laparoscope 
and real image of the subject. A number of IRILS transport the image to the eyepiece containing a magnifying lens. In the Hopkins rod-lens system, light is transmitted through glass columns and refracted through intervening air lenses. The camera is attached to the eyepiece of the laparoscope for processing.

Digital laparoscopes, in which the laparoscope and camera head are a single unit with the imaging sensor at the end of the laparoscope, have been available since the early 1990s. This (chip on stick technology) has been introduced in which CCD (Charge-coupling device-chip) will be at the tip inside the abdominal cavity. It is proved that the resolution of picture will be more than $250 \mathrm{k}$ pixels, but suffered from the inability to focus clearly at close distances to the tissue and simply never really caught on (Fig. 2.25).

\section{Telescope Fiber Bundle}

The telescope also contains parallel optical fibers bundle that transmit light into the abdomen from the light source via a light cable attached to the side of the telescope. The fiber bundle in the laparoscope and the fiber-optic light cable must be in excellent working order so as to achieve an optimal well-lighted picture. The fiber bundle located along a tract on the periphery of the telescope, and occupies less than half of the circumference of the telescope. It is exit at the inner tip of the telescope is corresponding to the attachment of the light cable to the side of the telescope.

\section{Laparoscopic Camera}

First medical camera was introduced by Circon Corporation in 1972. Laparoscopic camera is one of the very important instruments and should be of good quality (Fig. 2.26). Laparoscopic camera available is either of single chip or three chips. We all know that there are three primary colors (Red, green and blue). All the colors are mixture of these three primary colors in different proportion.

The (CCD) Charge-coupling device (Chip) is an electronic memory that records the intensity of light as a variable charge. Widely used in still cameras, camcorders and scanners to capture images, CCDs are analog devices. Their charges equate to shades of light for monochrome images or shades of red, green and blue when used with color filters. Three chips camera uses three CCDs, one for each of the red, green and blue colors (Fig. 2.27).

\section{Why Coupled?}

The "coupled" in the name is because the CCD is comprised of an array of imaging pixels and a matching array of storage pixels that are coupled together. After the imaging array is exposed to light, its charges are quickly transferred to the

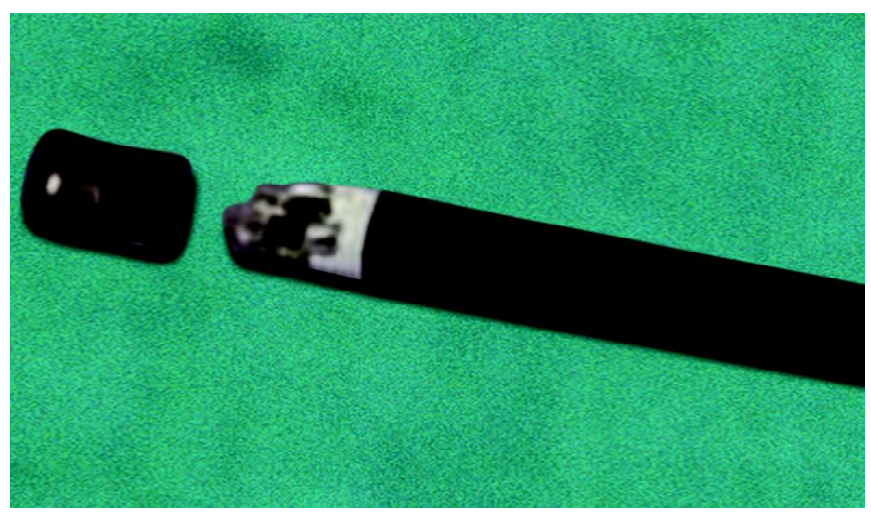

Fig. 2.25: Chip on stick technology

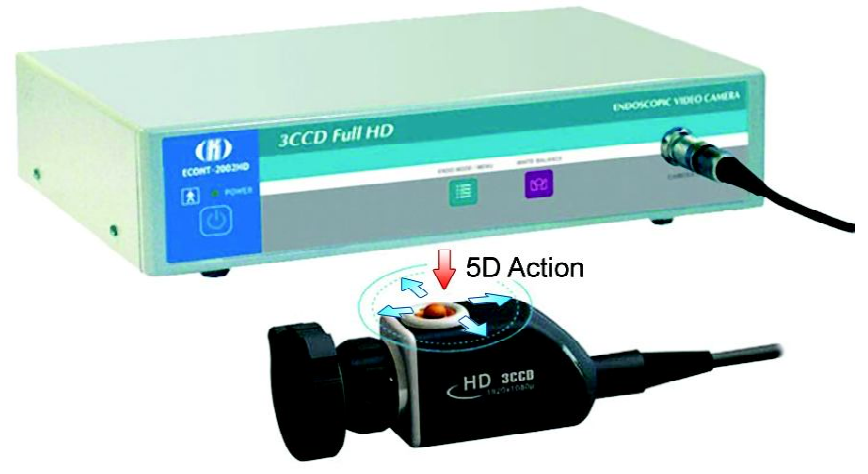

Fig. 2.26: Laparoscopic camera

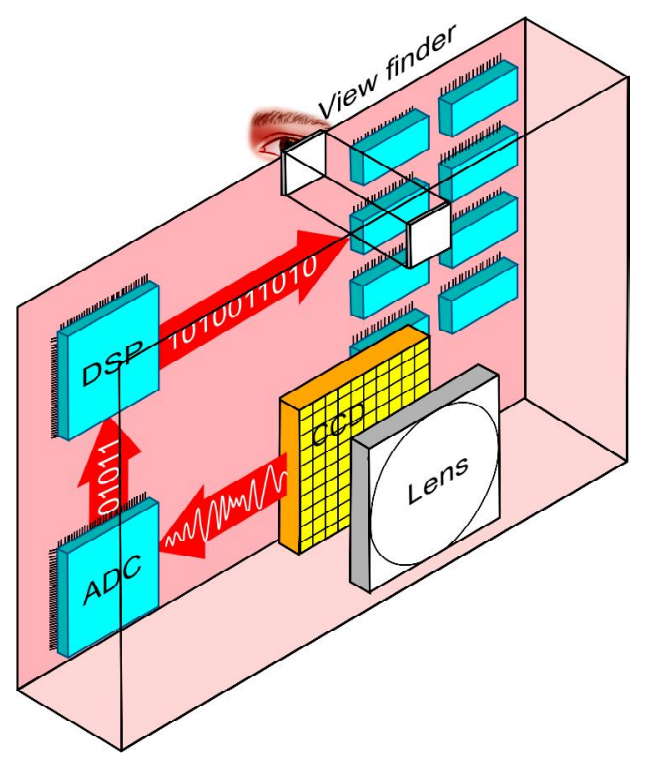

Fig. 2.27: In a camera, CCDs take the place of film. They are exposed to light, recording the intensities, or shades of light as variable charges. In the digital camera above, the variable, analog charges in the CCD are converted to binary data(0/1) by analog-to Digital converter chip (ADC) .DSP=Digital single processing 
storage array. While the imaging CCDs are being exposed to the next picture, the storage CCDs from the last picture are being read out a row at a time to the analog-to-digital converters (A/D converters) that transform the charges into binary data( $0 / 1$ ) to be processed (Fig. 2.28).

The camera system has two components: The head of the camera (Fig. 2.29), which is attached to the ocular of the telescope, and the controller, which is usually located on the trolley along with the monitor.

Within the head of camera is an objective zoom lens that focuses the image of the object on the chip, and a CCD chip that "sees" an image taken by telescope (Figs 2.30A and $B$ ). All modern miniature cameras used in minimal access surgery are based on the (CCD) charged couple device (chip). The CCD then converts optical image into an electrical signal that is sent through the camera cable to CCU (Camera control unit). The chip has light sensitive photoreceptors that generate pixels by transforming the incoming photons into electronic charges. The electronic charges are then transferred from the pixels into a storage element on the chip. A subsequent scanning at defined time intervals results in a black and white image with graey tones.

Pixel: PIX [picture]+ EL[element],picture element is the smallest element of a light-sensitive device, such as cameras that use charge-coupled devices. It is the smallest resolved
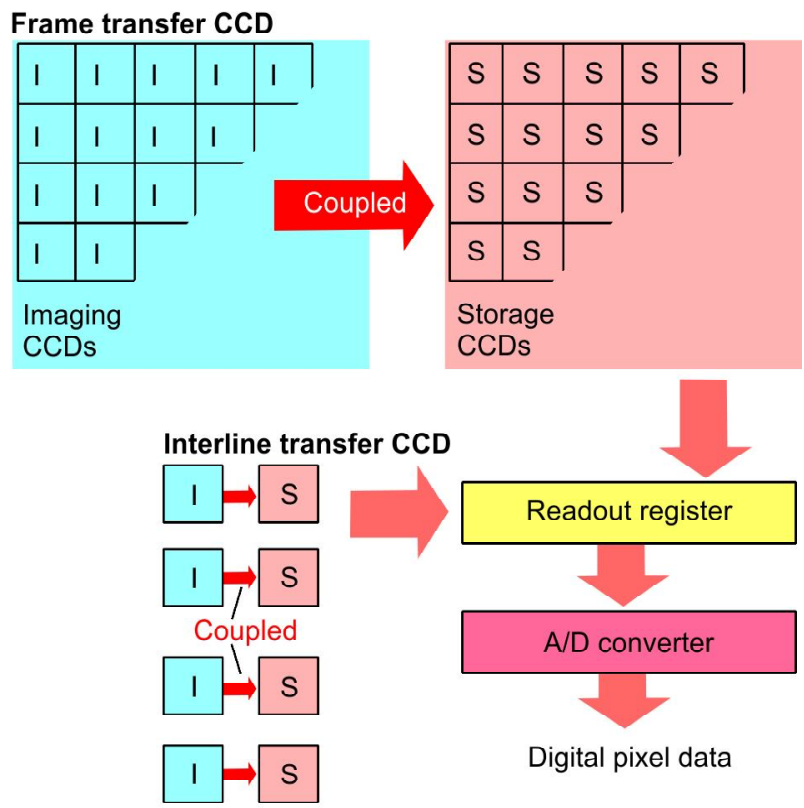

Charge-coupling methods

Fig. 2.28: The storage CCDs are either a separate array (frame transfer) or individual photosites (interline transfer) coupled to each imaging photosite. The charges can be transferred faster with the interline method because each storage component is closer to its imaging counterpart

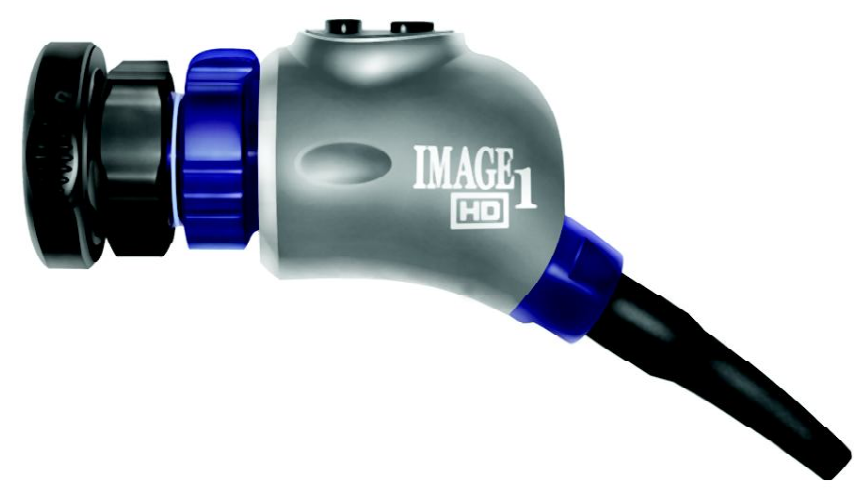

Fig. 2.29: Head of camera (CCD)

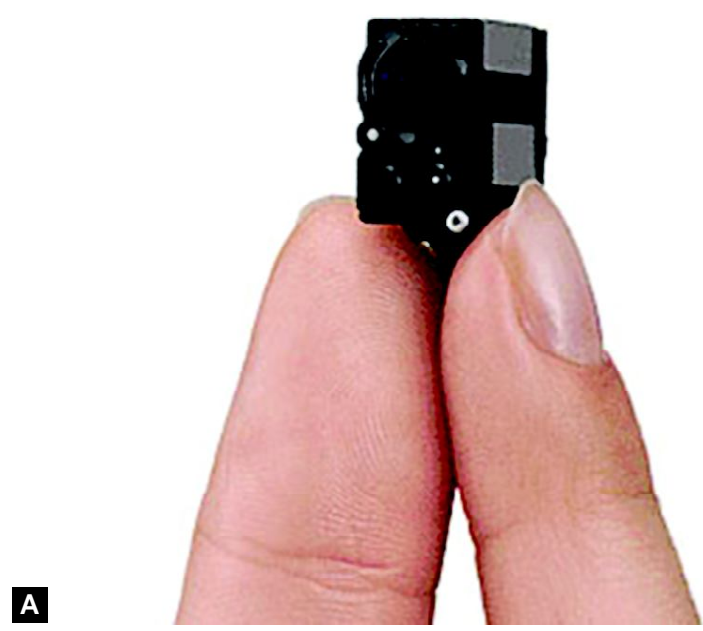

Frame transfer CCD

\begin{tabular}{|c|c|c|c|c|c|}
\hline I & I & I & I & I & $S$ \\
\hline I & I & I & I & & \\
\hline I & I & I & & & \\
\hline I & I & & & & \\
\hline & & & & & \\
\hline
\end{tabular}

Interline transfer CCD
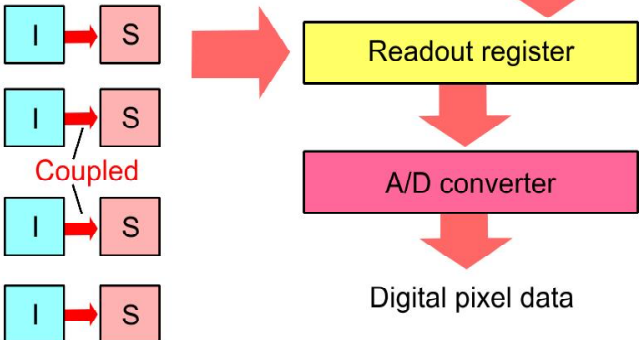

Digital pixel data

B

Figs 2.30A and B: CCD camera 
unit of a video image that has specific luminescence and color. Its proportions are determined by the number of lines making up the scanning raster (the pattern of dots that form the image) and the resolution along each line. In the most common form of computer graphics and the CCD devices, the thousands of tiny pixels that make up an individual image are projected onto a display screen as illuminated dots that from a distance appear as a continuous image. An electron beam creates the grid of pixels by tracing each horizontal line from left to right, one pixel at a time, from the top line to the bottom line.

The number of pixels determines the resolution. Screen resolution is rated by the number of horizontal and vertical pixels; for example, $1024 \times 768$ means 1,024 pixels are displayed in each row, and there are 768 rows (lines). Likewise, bitmapped images are sized in pixels: a $350 \times 250$ image has 350 pixels across and 250 down.

Pixels and Subpixels: In monochrome systems, the pixel is the smallest addressable unit. With color systems, each pixel contains red, green and blue subpixels, and the subpixel is the smallest addressable unit for the screen's electronic circuits. On a display screen, pixels are either phosphorus or liquid crystal elements. For monochrome, the element is either energized fully or not. For gray scale, the pixel is energized with different intensities, creating a range from light to dark. For color displays, the (RGB) red, green and blue subpixels are each energized to a particular intensity, and the combination of the three color intensities creates the perceived color to the eye.

The average chip contains 250000 to 380000 pixels.

Cameras are classified according to the number of chips. These differ among other things, in the way they relay color information to the monitor. Color separation is used to create a colored video image from the original black and white. In single chip cameras, color separation is achieved by adding a stripe filter that covers the whole chip. Each stripe accepts one of the complementary colors (magenta, green, cyan, or yellow) and each pixel is assigned to one stripe.

In single chip camera, these three primary colors are sensed by single chip. In three chip camera, there are 3 CCD chips for separate capture and processing of 3 primary colors(R, G, B colors). In three chip cameras color separation is achieved with a prism system that overlies the chips. Each chip receives only one of the three primary colors (Red, green or blue). This system gives a higher resolution and better image quality because the pixel number is three times greater.

The video information, color and light are scanned at a rate of 525 lines per frame and 30 frames per second. Picture resolution determines the clarity and detail of the video image. Higher the resolution, the better will be quality of image. The resolution of picture is ascertained by the number of distinct vertical line that can be seen in the picture. The higher the resolution numbers, the sharper and cleaner image will form. The CCU of camera is connected with monitor and monitor converts the electrical image back to the original optical image.

These 3 chip camera has unprecedented color reproduction and highest degree of fidelity. Three chip cameras have high horizontal image resolution of more than 750 lines.

\section{Chip on Stick Technology}

Currently chip on stick technology has been introduced in which CCD will be at the tip inside the abdominal cavity. It is proved that the resolution of picture will be more than $250 \mathrm{k}$ pixels (Fig. 2.26).

\section{Focusing of Laparoscopic Camera}

Laparoscopic camera need to be focused before inserting inside the abdominal cavity. At the time of focusing, it should be placed at a distance of approximately $10 \mathrm{~cm}$ away from the target for the $10 \mathrm{~mm}$ telescope, $5 \mathrm{~mm}$ for $5 \mathrm{~mm}$ telescope and $4 \mathrm{~mm}$ for $4 \mathrm{~mm}$ telescope, with an average distance of approximately $5 \mathrm{~mm}$ for all telescopes. This distance is optimum for focusing because at the time of laparoscopic surgery, most of the time we keep the telescope at this distance.

\section{White Balancing of Camera}

White balancing should be performed before inserting camera inside the abdominal cavity. White balancing is necessary every time before start of surgery because every time there is some added impurities of color due to following variables:

- Difference in voltage

- Different cleaning material used to clean the tip of telescope which can stain the tip

- Scratches, wear and tear of the telescopes eye-piece, object piece and CCD of camera.

White balancing is done by keeping any white object in front of telescope attached with camera that senses white object as reference. It adjusts its primary color (Red, blue and green) to make a pure natural white color.

\section{Laparoscopic Video Monitor}

Surgical monitors are slightly different from the TV which we watch at home (Fig. 2.31). Monitor lasts long so a surgeon gets high end product with at least 600 lines resolution. The size of the screen varies from 8 to 21 inches. The closure the surgeon is to the monitor, the smaller the monitor should be to get better picture. The basic principle of image 
reproduction is horizontal beam scanning on the face of the picture tube. This plate is coated internally with a fluorescent substance containing phosphorus. This generates electrons when struck by beams from the electron gun. As the beam sweeps horizontally and back, it covers all the picture elements before reaching its original position. This occurs repetitively and rapidly. This method is called 'H orizontal linear scanning'. Each picture frame consists of several such lines depending on the type of system used.

\section{Endoscopic Vision (Video) Technology Evolution}

In the past endoscopic procedures were done without the aid of monitors. The operator visualized the interiors of the patient directly through the eyepiece of the scope. This method was associated with many difficulties. He was the only person who could observe the procedure leading to poor coordination with other members of the team. As a result extensive and difficult procedures could not be performed. The magnification was very poor. Surgeons had to face problems with posture leading to discomfort and strain as his eye was always glued to the eyepiece. He had difficulties in orientation due to visualizing with only one eye.

As better methods of communication developed the introduction of television brought about a significant impact. A good magnification of the image was reproduced. All members of the team could visualize the procedure. Surgeons could operate more comfortably. Complex procedures began to be undertaken and were even recorded.

Soulas in France first used television for endoscopic procedures in 1956. He demonstrated the first televised bronchoscopy. A rigid bronchoscope was attached to a black and white camera that weighed about $100 \mathrm{lbs}$.

In 1959, a laparoscopic procedure was demonstrated using a closed circuit television program using the "Fourestier method". This method was developed by transmitting an intense beam of light along a quartz rod from the proximal to distal ends of the laparoscope.

The first miniature endoscopic black and white television camera was developed in Australia in 1960 (Fig. 2.32). It weighed 350 grams, was $45 \mathrm{~mm}$ wide and $120 \mathrm{~mm}$ long. Because of its small dimensions it could be attached to the eyepiece.

\section{TELEVISION SYSTEMS}

The existing television systems in use differ according to the country. The USA uses the NTSC (National Television System Committee) system. In European countries the PAL (Phase alternation by line) system is in use. There is also a French system called SECAM (SEquential Color and Memory). The broadcasting standards for each are summarized in Table 2.2 .
The final image depends upon the number of lines of resolution, scanning lines, pixels and dot. pitch. The number of black and white lines a system can differentiate gives the lines of resolution. These can be horizontal and vertical. Horizontal resolution is the number of vertical lines that can be seen and vice versa. Pixels denote the picture elements and they are responsible for picture resolution. The more

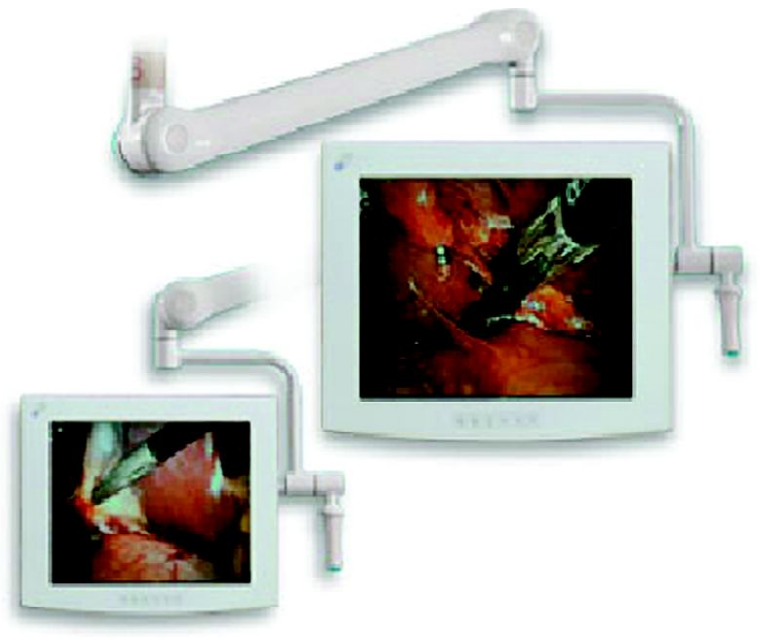

Fig. 2.31: Laparoscopic monitor

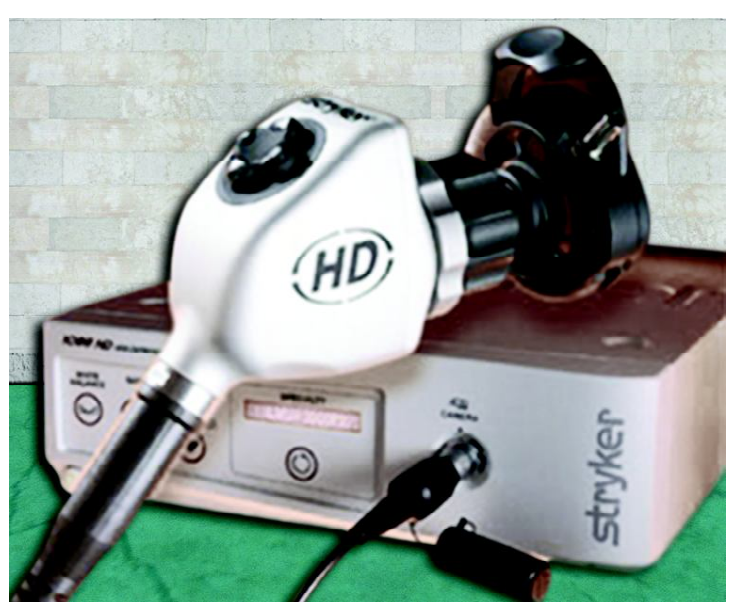

Fig. 2.32: Endoscopic camera

Table 2.2: Different types of monitor systems

\begin{tabular}{llll}
\hline System & PAL & SECAM & NTSC \\
\hline Number of lines & 625 & 625 & 525 \\
Visible lines (max) & 575 & 575 & 486 \\
Field frequency cycles (cps) & & & \\
per second & 50 & 50 & 60 \\
Frames per second & 25 & 25 & 30 \\
\hline
\end{tabular}


number of pixels is, the better the resolution. They are represented on the camera chip by an individual photodiode. The restricting factor of information on a scan line is the 'dot pitch' that represents phosphorus element size.

The NTSC system has certain drawbacks. N ot all the lines of resolution are used. The maximum number of lines visible is reduced by 40 . Improving the resolution of the camera will not improve monitor resolution. This is due to a fixed vertical resolution. In addition to these problems, if the phase angle is disturbed even a little, it produces unwanted hues.

The PAL system is superior in certain aspects. It can overcome this problem by producing alternations over the axis of modulation of the color signed by line. This system also deals with problems of flickering. It involves a process called 'interlacing' where odd and even lines in a field are scanned alternatively.

The SECAM system is similar to PAL in these aspects except that the signals are transmitted in sequence.

\section{The Formation of the Color Image}

Another important aspect one has to keep in mind is the formation of the color image. This is done by super-imposing the data for color on the existing black and white picture. The black and white signal is monochromatic signal, and combines with the composite color signal. This gives the final color signal.

Luminance (brightness) is delivered by the black and white signal. Chrominance (color) is delivered by the color signal. It is called composite as it contains the three primary color information (Red, Blue and Green). A system that combines luminance and chrominance into one signal is called a "compound system".

Color values can be problematic as they can go out of phase. This is due to their high sensitivity. Applying a reference mark for the signal on the scanning line called as "color burst" can prevent this. The color on a monitor can be calibrated. This can be done manually by using the standard color bars of NTSC or by using other methods like "blue gun". N ew monitors do not require this, as calibration can be done automatically.

\section{Monitor Connecting Cables}

Images cannot be visualized on the monitor unless they are wired. Monitor cables are of three types. The RGB cable has 3 wires, one for each primary color. The $Y / C$ cable has 2 wires, one for the luminance $(Y)$ and one for the chrominance $(\mathrm{C})$ component. The composite cable consists of one pair of wires. An important factor to realize is that no matter what type of cable is used, whether it has better bandwidth or other advantages, the final resolution depend upon the monitor used.

\section{Frames of Reference in Vision}

We face many problems with monitors in regard to minimal access surgery. But before dealing with them, a mention of the frames of reference in vision would be apt. NJ Wade's paper on 'Frames of reference in vision' mentions various frames namely retinocentric, egocentric, geocentric and pattern centric. He applies these to minimal access surgery and finds a dissociation of pattern centric motion (seen on the monitor) and the area of manipulation. Any visual motor task requires a match between the coordinate systems operating in both vision and motor control. Knowledge of these frames can alter our perspective of the way things happen in minimal access surgery with respect to vision.

\section{Drawbacks with the Monitor}

After routine use, we encounter many drawbacks with the monitor.

Only a 2D image can be seen on present day monitors.

The operative field is represented only by monocular depth cues.

Monitor positioning is such that the visual motor axis is disrupted.

The monitor distance from the surgeon is also quite far. As a result, the efficiency of the surgeon decreases.

A part from pictorial depth cues the picture can be further disturbed by anticues. These may originate from the monitor. Glaring effect due to reflection is one of these important anticues.

\section{Imaging Technical Terms}

Resolution means the degree of sharpness of a displayed or printed image; simply, the ability of a television or film image to reproduce fine detail.

Resolution is defined as (Pixels per inch); the maximum number of pixels that can be displayed on a screen monitor, expressed as (number of horizontal pixels) $\times$ (number of vertical pixels), i.e. $1024 \times 768$. The ratio of horizontal to vertical resolution is usually $4: 3$, the same as that of conventional television sets.

For a monitor, a screen resolution of $1920 \times 1200$ means 1,920 pixels horizontally across each of 1,200 lines, which run vertically from top to bottom.

For printers and scanners, resolution is expressed as the number of dots per linear inch (Printed dots per inch- dpi). 300 dpi means $300 \times 300$ or 90,000 dots per square inch. Laser printers and plotters have resolutions from 300 to $1,200 \mathrm{dpi}$ and more, whereas most display screens provide less than 100 dpi (Figs 2.33 and 2.34).

Gain controls the brightness of the image under conditions of low light by recruiting pixels to increase signal strength. Clearly, this step results in some loss of image 
resolution. This increases light but results in a grainy picture with poorer resolution. It also may create a loss of color accuracy owing to amplification of the noise-to-signal ratio).

The gain should be off at the beginning of a routine procedure (not needed in routine situations). There are two good reasons for this. First, inadequate light at the beginning of the procedure is an indication that a piece of equipment is malfunctioning, a situation that should be rectified before getting too involved with the procedure. Second, the use of "gain" comes at the expense of picture resolution, resulting in a somewhat grainy image on the monitor, while turning the brightness knob to maximum produces a very washedout picture. A good general rule of thumb to follow is to look in the right upper quadrant and visualize the space over the liver. The entire dome of the liver and right lateral diaphragm should be well lit and easily seen. If not, then the lighting system should be evaluated and optimized prior to beginning the dissection (Figs 2.35A and B).

Contrast: The extent to which adjacent areas of an optical image, on a monitor screen, differ in brightness. It is the difference in visual properties that makes an object (or its representation in an image) distinguishable from other objects and the background. In visual perception of the real world, contrast is determined by the difference in the color and brightness of the object and other objects within the same field of view. Because the human visual system is more sensitive to contrast than absolute luminescence, we can perceive the world similarly regardless of the huge changes in illumination over the day or from place-to-place (Fig. 2.36).

Luminescence, is a general term applied to all forms of cool light, i.e. light emitted by sources other than a hot, incandescent body. It is a process by which an excited material emits light in a process not caused solely by a rise in temperature. The excitation is usually achieved with, ultraviolet radiation, $\mathrm{X}$-rays, electrones, alpha particles, electrical fields, or chemical energy. The color or wavelength, of the light emitted is determined by the material, while the intensity depends on both the material and the input energy. Examples of luminescence include light emissions from neon lamps, luminescent watch dials, television and computer screens, fluorescent lamps, and fireflies.

\section{Modulation Transfer Function}

The endoscopes transmit resolution and contrast to the monitor.The efficacy by which this occurs determines the more delicate aspects of the image. Resolution and contrast can be measured on a especially designed optical bench and expressed as modulation transfer function (MTF). If there is excessive glare in the picture then contrast and resolution decrease. Distortions of the image can occur and if these lines seem to curve outwards they are called "barrel
STANDARD SCREEN RESOLUTIONS

These resolutions have a 1.33:1 (4:3) aspect ratio, except for SXGA, which is $1.25: 1$.

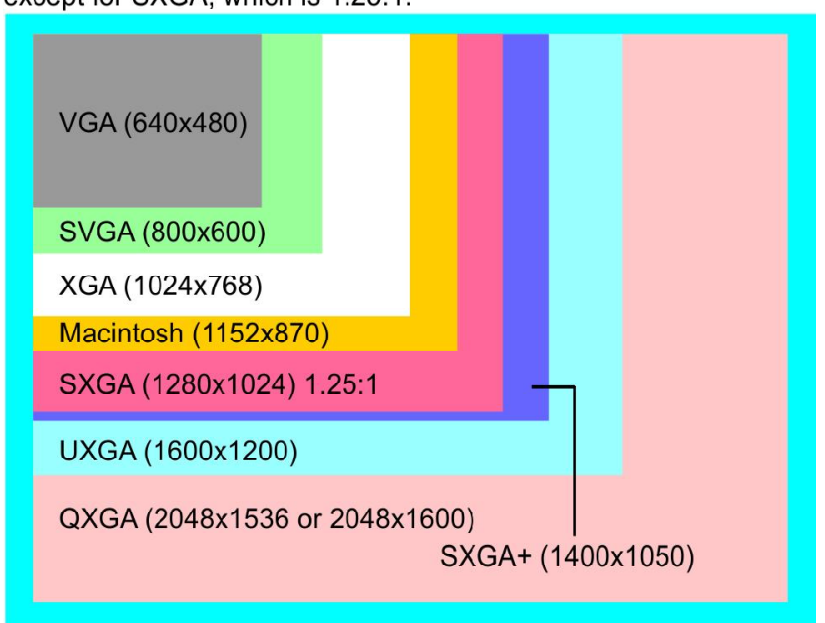

Fig. 2.33: Standard screen resolution,(4:3) aspect ratio,i.e.the height is somewhat approxamates to the width

WIDE SCREEN RESOLUTIONS

HDTV uses a 1.78:1 (16:9) aspect ratio.

Many hollywood movies are shot at 1.85:1 and 2.35:1.

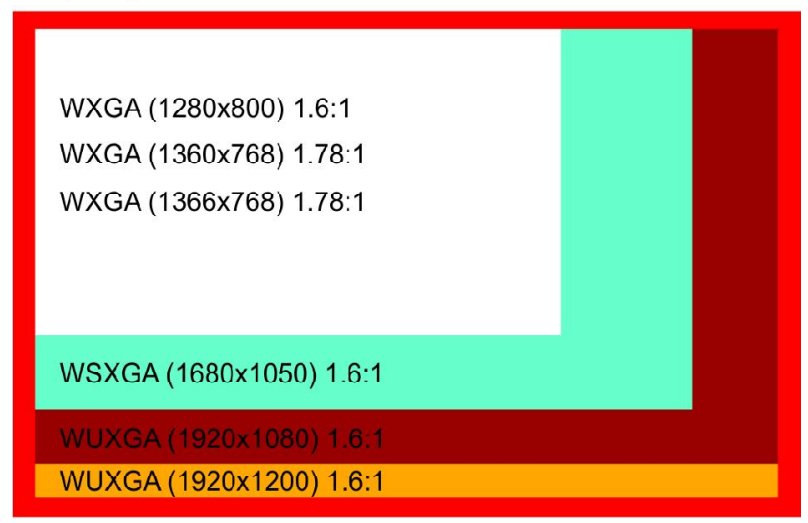

\section{LARGER WIDE SCREENS}

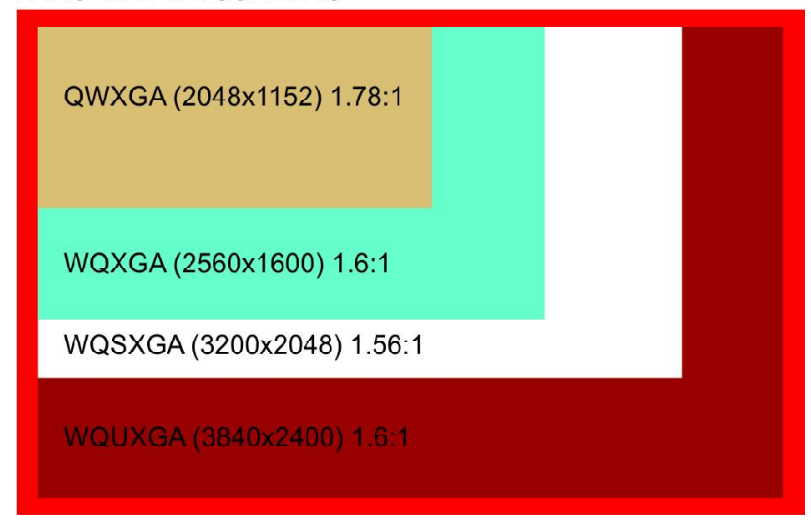

Fig. 2.34: WIDE \& LARGER Screen Resolution, (16:9) aspect ratio,i.e. the width is more than the height 

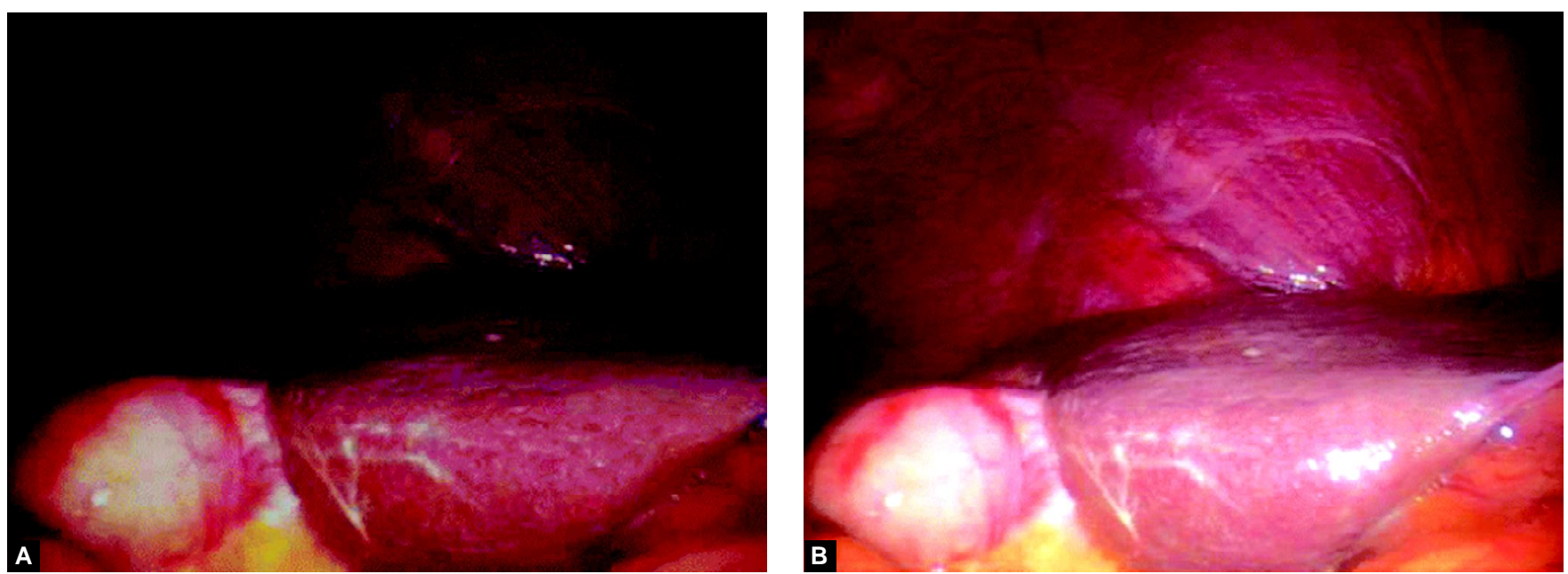

Figs 2.35A and B: Gallbladder lighting scene: (A) Poorly lit right upper quadrant. The diaphragm is barely visible; (B) Same patient after faulty light cable is replaced
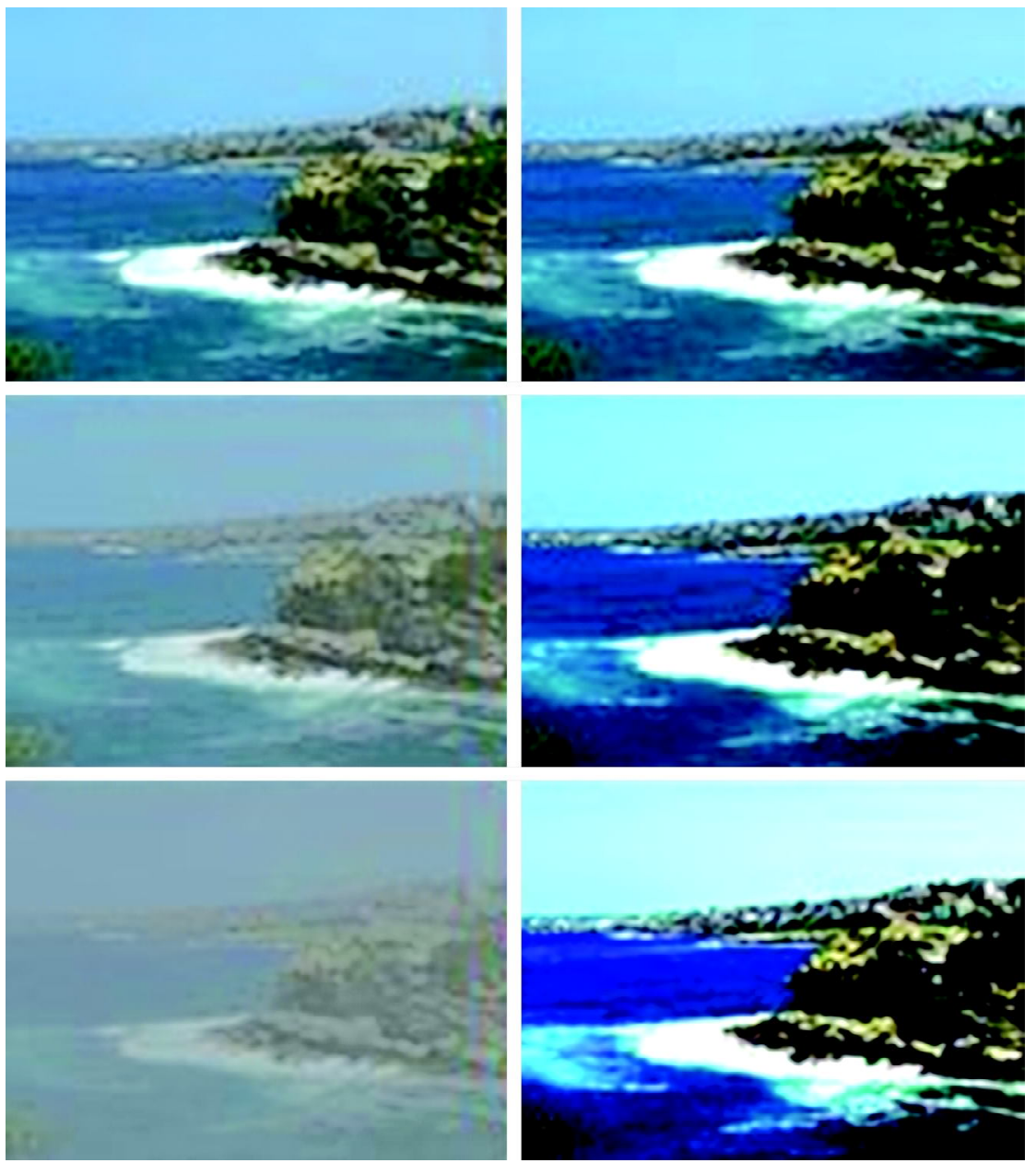

Fig. 2.36: Changes in the amount of contrast in a photo, left side of the image has low contrast, the right side of the image has higher contrast 
distortion". Field curvature occurs when there is improper focus of the center from other parts. Astigmatism can occur when some lines of different orientation are present in focus and others are not.

\section{Temporal Aliasing}

When a moving object is shown on a monitor, unless the speed with which it is moving is similar to the refresh rate, then jerky movements will occur. This is called "temporal aliasing". This can be prevented by the use of filters, or by performing slow movements. Fatigue and headache can occur due to disturbance of saccadic eye movements. These are rapid eye movements used to visualize the borders of a field.

\section{Gaze-Down Position}

When a surgeon has to constantly look in a different direction and operate in another his efficiency to perform declines. The job becomes even more difficult if the monitor is positioned at a further distance, giving rise to spatial disorientation. A surgeon can perform optimally if he can look and operate in the same direction as in open surgery. This can also be called the "gaze-down position".

\section{THREE-DIMENSIONAL VISION}

One of the major limitations of minimal access surgery is the loss of depth perception. The surgeon works with an artificial two-dimensional video pictures available on the monitor. There is a need to develop some mechanism to improve depth perception or stereoscopic vision. Stereoscopic vision is needed for precise and fast complex manipulations because perception of space and depth are necessary in surgery.

\section{Stereopsis: (3D Perception)}

Stereopsis refers to perception of three-dimensional shape from any source of depth information. Unlike horses, humans have two eyes located side-by-side in the front of their heads. Due to the close side-by-side positioning, each eye takes a view of the same area from a slightly different angle (Fig. 2.37).

The two eyes have different views of the visual world and these different views set-up disparities that give us information about relative depth of the image that is third dimension of vision. Binocular disparity results when image of an object falls on different areas of the two retinas and binocular vision or stereopsis is impression of depth resulting from differences in the images on these two retinas. We lose stereoscopic vision in laparoscopy because both eyes see same two-dimensional pictures available on the monitor.

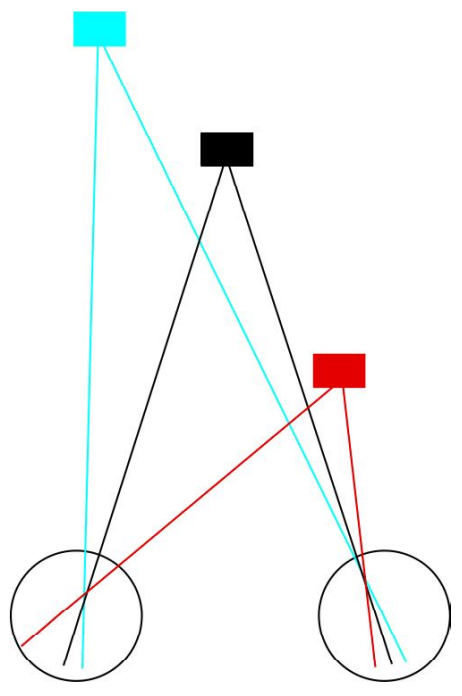

Fig. 2.37: Difference in angle of light on different retinas

Stereoscopic vision is simultaneous vision with two eyes (B inuclear vision), producing a visual experience of the thirddimension (the depth), that is, a vivid perception of the relative distances of objects in space. In this experience the observer seems to see the space between the objects located at different distances from the eyes (The three-dimensions are width, length, and depth).

It is present in normal binocular vision because the two eyes view objects in space from two points, so that the retinal image patterns of the same object points in space are slightly different in the two eyes. The stereoscope, with which different pictures can be presented to each eye, demonstrates the fundamental difference between stereoscopic perception of depth (third dimension) and the conception of depth and distance from the monocular view.

Humans normally have Binocular vision, i.e. separate images of the visual field are formed by each eye; the two images fuse to form a single impression. Because each eye forms its own image from a slightly different angle, a stereoscopic effect is obtained, and depth, distance, and solidity of an object are appreciated. Stereoscopic color vision is found primarily among the higher primates, and it developed fairly late on the evolutionary scale.

\section{Binocular Vision}

Vision with two eyes. In binocular vision, the visual axes of the eyes are arranged in such a manner that the images of the object viewed strike the identical portions of the retinas of both eyes. This produces a single stereoscopic image-a view of the world in relief. Binocular vision also makes it possible to determine visually the relative location of objects in space and to judge their distance from each other. When 
looking with one eye- that is, with monocular vision - the distance of objects can likewise be judged, but not as accurately as with binocular vision.

\section{Physiology of Three-dimensional Vision}

Human eye is sensitive to the electromagnetic wavelength between 400 and $700 \mathrm{~nm}$. The "electromagnetic energy" in the range of approximately "400 to 700 nm", which the human eye can transduce, is called "light". The eyes transduce light energy in the electromagnetic spectrum into nerve impulses.

In 1838, Charles Wheatstone published the first paper on stereopsis entitled "On some remarkable and hitherto unobserved, phenomena of binocular vision". In this he pointed out that the positional differences in the two eye's images due to their horizontal separation yielded depth information. Prior to this time, the principal problem in the study of binocular vision was "how the world seen as single when we have two different views of it?" People thought that only objects with the same visual directions, falling on corresponding points would be seen as single, all other points would be double. By means of the stereoscope that he invented. Wheatstone demonstrated that the stimulation of noncorresponding points yield singleness of vision, and results in the Perception of Depth.

Cells in visual cortex are sensitive for binocular vision. Some cells are sensitive for corresponding areas of the left and right retinas and some have sensation for noncorresponding areas of the two retinas. The difference in image on the two retinas of eye is perceived by cortex as a Depth. Binocular disparity provides the visual system with information concerning the three-dimensional layout of the environment. Recent physiological studies in the primary visual cortex provide a successful account of the mechanisms by which single neurons are able to signal disparity.

Recent studies of visual perception have begun to reveal the connection between neuronal activity in the brain and conscious visual experience. Transcranial magnetic stimulation of the human occipital lobe disrupts the normal perception of objects in ways suggesting that important aspects of visual perception are based on activity in early visual cortical areas. Recordings made with microelectrodes in animals suggest that the perception of the lightness and depth of visual surfaces develops through computations performed across multiple brain areas.

Even though the picture on the monitor istwo-dimensional, the operator can assume some depth due to different cues for monocular vision. This is known as "Depth cue" (Fig. 2.38).

Among the entire depth cue mentioned above, only few monocular are available on the monitor screen. Oculomotor binocular depth cue like convergence, accommodation and stereopsis is lost in two-dimensional monitor screen.
Even though the picture is two-dimensional, these depth cues helps our brain to interpret a two-dimensional image into virtual three-dimensional feeling. Today, the picture on the screen is 2D but our brain is continuously converting this 2D image in 3D by the help of different monocular depth cues. This conversion of two-dimensional pictures into threedimensional pictures by brain helps the surgeon to perform a task in laparoscopic surgery.

A telescope with light delivering through a separate illumination cannula was developed by Schurr in 1996 (Fig. 2.39).

With slight modification, the section for minimally invasive surgery, University of Tübingen, and MGB Endoskopische Geräte GmbH Berlin Company have introduced a new shadow telescope in 1999 (Fig. 2.40).

Shadow telescope is a rigid $10 \mathrm{~mm}$ endoscope with $30^{\circ}$ view direction and uses additional illumination fibers ending in an optimized distance behind the front lens. This arrangement of illumination fibers creates a more natural and more plastic appearance, a better balanced contrast and a well-dosed visible shadow. The shadow gives

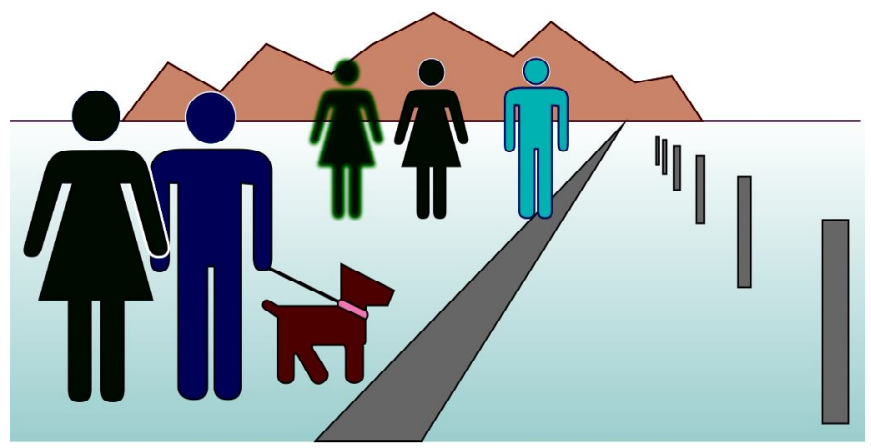

Fig. 2.38: Different depth cues: The depth cues which are crossed are lost in minimal access surgery

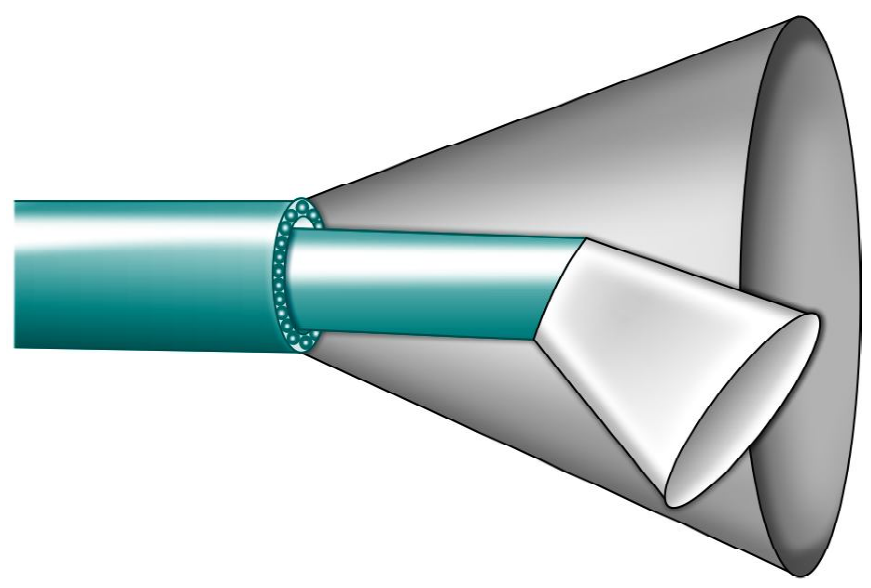

Fig. 2.39: Shadow telescope by Schurr 
additional secondary space clues and, therefore, improves orientation and judgment of the three-dimensional properties.

In shadow telescope, angle between the first and second source of illumination is fixed and due to this shadow is also fixed. The second problem with this telescope is that the light which is creating shadow comes from below but we do not usually experience getting illuminated from the floor. All the light in our day-to-day life is coming from above, and most of the time, the shadow we are seeing lies below the object. But in this telescope, the shadow is above the object and so the shadow seems to be unnatural.

\section{INVENTION OF IDEAL SHADOW IN LAPAROSCOPIC SURGERY}

The first ideal shadow was introduced in laparoscopic surgery by Dr RK Mishra in the University of Dundee, UK (Fig. 2.41). This is based on the introduction of one additional light source through a separate port for the generation of natural shadow in minimal access surgery.

Due to the limitations of currently present shadows producing techniques, necessity was felt for development of some newer methods of shadow production. Shadow can play a very powerful role in defining form by giving the object a three-dimensional feel and it is easy to generate. Although shadow is an important depth cue, but too much shadow may cause blurred working field for surgeons and shadow in wrong direction may have adverse effect on the performance of surgeon.

To increase the task performance in minimal access surgery we recommend certain general rules about shadows. First, light which will cast shadow ideally should come from above. Second, to enhance the task performance with shadow, the contrast of shadow should be mild (22-42\%). This useful percentage of contrast can be achieved by equal intensity setting and equal distance of both the light sources from the operating field. Third, too much shadow (more than $60 \%$ ) should be avoided because very dark shadow can increase the error rate by interfering with the view of plane of dissection.

\section{THREE-DIMENSIONAL VIDEO SYSTEMS}

\section{The 3D Technology Systems}

The most commonly used systems depend on rapid time sequential imaging with two cameras and one monitor and are based on the physiological phenomenon of retinal persistence. Both channels alternate (open/close) with sufficient speed $(50-60 \mathrm{~Hz}$ ) to avoid detection of flicker by the human eye. The monitors must therefore have double the frequency $(100-120 \mathrm{~Hz})$. Sequential switching between the two eyes is necessary to ensure that the correct image (left and right) falls on the corresponding retina otherwise picture will overlap. This is achieved by wearing special optical glasses that act as alternating shutters to each eye. The current problem with these optical shutters, especially the active battery operated liquid crystal display type shutter is loss of brightness and color degradation. There is no clinically proved evidence that current three-dimensional systems improve performance of laparoscopic surgery.

The current 3D technology is harmful to the surgeon on prolonged use as it gives incorrect depth perception and results in headache and eyestrain.

Another way in which 3D images can be obtained is a mechanism by which the surgeon wears a polarized glass. The shutter mechanism is present in the monitor. The final image, however, occurs by the fusion of the two images in

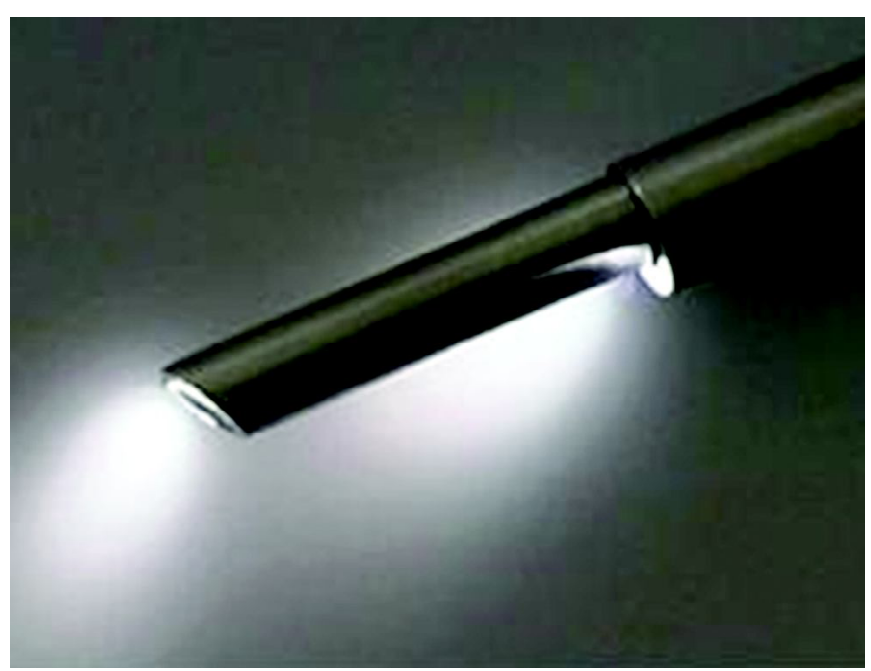

Fig. 2.40: Shadow telescope of Tübingen University

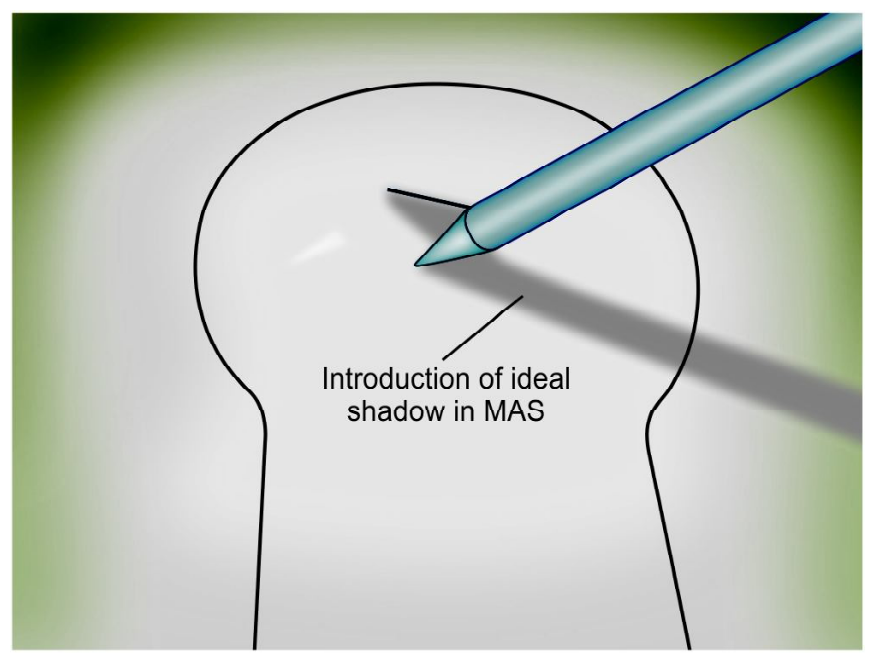

Fig. 2.41: Shadow in laparoscopy 
the brain. The current 3D systems can only be operated from a very close distance and if placed further will not produce the desired 3D effect.

\section{New 3D Techniques}

With the increasing demands for technological development many new techniques are currently under trial. These seem to eliminate some of the problems encountered, but only time and repeated use will tell.

Head Mounted Display (HMD) is an interesting technique that aims at normalizing the visual-motor axis. It consists of a monitor and the necessary connections mounted to the surgeon's head with the power supply pack attached to the back of the surgeon's shirt. It is not very heavy and also allows the surgeon to view peripherally.

The optical characteristics are:

- Lines of resolution-420 × 320 lines

- Contrast ratio- 100: 1

- Horizontal field of vision-22 2

- Diagonal field- $27.5^{\circ}$

- Vertical field-19\%.

The surgeon using the display will have to make adjustments to the inter-pupillary distance, focus and the distance from his eyes each time. Studies have shown HMD to have certain advantages. It is light weight, comfortable to position, reduces mental stress, is cheaper than monitor systems, and decreases eye strain. It allows the surgeon to visualize the operative field directly (the abdomen and ports). The problems, however, are that the picture is granular, definition is not very good and nausea can occur.

As mentioned before, the gaze down position is said to improve the performance of the surgeon as it brings the alignment between his hands and eyes to normal. This principle has been used in a project called "view-site". This mechanism is used to project the operative field image onto a sterile screen placed on the patient's abdomen close to the original area of surgery. However, it cannot be used for extensive procedures as the image field is small, resolution is not up to the mark and separation and identification of tissue planes becomes difficult if bleeding were to occur.

Image display systems are now available which project the image on to a sterile screen overlying the chest of the patient. This aids both cerebral processing of the image and endoscopic manipulations and improves both quality and efficiency of performance (Fig. 2.42). The limitation of the current "gaze down" image display systems are diminished resolution and encroachment of the operative field by the sterile screen.

\section{Suspended Image System}

An experimental system (suspended image system [SIS]) being developed at the University of Dundee and the Central

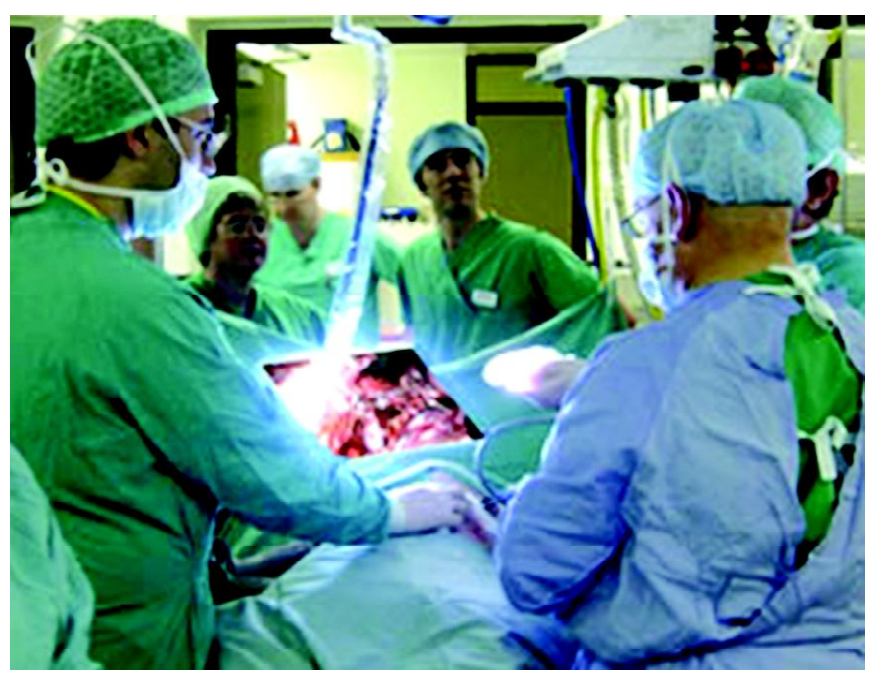

Fig. 2.42: Projection systems in minimal access surgery

Research Laboratory (EMI) projects the image in air on top of the patient, which means there is no screen to obstruct the surgeon's movement. This is called the suspended image system (SIS). It consists of two components: a high precision retro-reflector and a beam splitter. With the help of these, the system can produce images with good resolution and can suspend them on top of the patient in close vicinity to the operative site. The advantages of this method are that there is no distortion, object can be placed anywhere, focal length is not specific and the image is similar to the original in size. This system is also said to improve the sense of depth, as there are no anti-cues. Also, the visual motor axis is correctly aligned for optimal performance.

VISTRAL is a system currently under trial. The advantage of this system is that it does not allow flatness cues to occur in 2D pictures. This improves the sense of depth and it does not require binocular depth cues. It is also said to reduce fatigue and eye strain. However, this system does not bring about any changes to resolution, brightness and color.

\section{Advancement in TV System Technology}

\section{High Definition Television}

A nother remarkable advancement in technology is the High Definition Television (HDTV). It uses component signals, the resolution of the picture is much better, and there are no distortions. They use about 1,100 lines of resolution.

\section{Palplus}

The alternatives to HDTV are the PALPLUS which is an advanced modification of PAL and D2-MAC, HD-MAC which are used for satellite transmissions. 
Table 2.3: Different types of TV system

\begin{tabular}{lrcr}
\hline System & $\begin{array}{c}\text { Japan } \\
\text { (NHK/Sony) }\end{array}$ & $\begin{array}{c}\text { Europe } \\
\text { (EUREKA 95) }\end{array}$ & USA \\
\hline Number of lines & 1125 & 1250 & 1050 \\
Visible lines (92\%) & 1035 & 1150 & 966 \\
Pixels per line & 1831 & 2035 & 1709 \\
Total number of pixels & 1895085 & 2340250 & 1650894 \\
Field frequency (cps) & 60 & 50 & 59,94 \\
Luminance & 20 & 20 & 20 \\
Chrominance & 7 & 7 & 7 \\
\hline
\end{tabular}

These systems, however, require large amounts of space and can cause problems during transmission. Due to the increased definition, small unwanted movements can be magnified and visual stress can be increased.

Some systems are currently under evaluation and their requirements are given in Table 2.3.

\section{BIBLIOGRAPHY}

1. Berber E, Pearl J M, Siperstein AE. A simple device for measuring the resolution of videoscopic cameras and laparoscopes in the operating room. Surg Endosc. 2002;16: 1111-13; discussion 1114.

2. Berci G, Schwaitzerg SD. The importance of understanding the basics of imaging in the era of high-tech endoscopy: part I. Logic, reality, and Utopia. Surg Endosc. 2002;4:377-80.

3. Bhayani SB, Andriole GL. Three-dimensional (3D) vision: does it improve laparoscopic skills? An assessment of 3D headmounted visualization system. Rev Urol. 2005;7:211-4.

4. Byrn J C, Schluender S, Divino CM, et al. Three-dimensional imaging improves surgical performance for both novice and experienced operators using the da Vinci Robot System. Am J Surg. 2007;193:519-22.

5. Chan AC, Chung SC, Yim AP, et al. Comparison of two dimensional vs. three-dimensional camera systems in laparoscopic surgery. Surg Endosc. 1997;11:438-40.

6. Ganai S, Seymour NE. VR to OR for Camera Navigation. In: Westwood J D, et al. (eds), Medicine Meets Virtual Reality IOC press, Amsterdam. 2005;111(13):45-8.

7. Grantcharov TP, Bardram L, Funch-J ensen $P$, et al. Impact of hand dominance, gender and experience with computer games on performance in virtual reality laparoscopy. Surg Endosc. 2003;17:1082-5.

8. Haluck RS, Gallagher AG, Satava RM, Webster R, Bass TL, Miller CA. Reliability and validity of Endotower, a virtual reality trainer for angled endoscope navigation. Stud $\mathrm{H}$ ealth Technol Inform. 2002;85:179-84.

9. Hanna GB, Cuschieri A. Influence of two-dimensional and three-dimensional imaging on endoscopic bowel suturing. World J Surg. 2000;24:444-9.

10. Hanna GB, Shimi SM, Cuschieri A. Randomized study of the influence of two-dimensional versus three-dimensional imaging on performance of laparoscopic cholecystectomy. Lancet. 1998;351:248-51.

11. HartSG, Staveland LE . Development of a multi-dimensional workload rating scale. Results of empirical and theoretical research. In: Hancock PA, Meshkati N (eds), Human Mental Workload. Elsevier, Amsterdam. 1988;139-83.

12. Herron DM, Lantis J C, Maykel J, et al. The 3-D monitor and head-mounted display. A quantitative evaluation of advanced laparoscopic viewing technologies. Surg Endosc. 1999;13:751-55.

13. Jones DB, Brewer JD, Soper NJ. The influence of threedimensional video systems on laparoscopic task performance. Surg Laparosc Endosc. 1996;6:191-7.

14. Korndorffer J R J r, Hayes DJ, Dunne J B, Sierra R, Touchard CL, Markert RJ, Scott DJ. Development and transferability of a cost-effective laparoscopic camera navigation simulator. Surg Endosc. 2005;19:161-7.

15. Korndorffer J R J r, Stefanidis D, Sierra R, Clayton J L, et al. Validity and reliability of a videotrainer laparoscopic camera navigation simulator. Surg Endosc. 2005;19:S246.

16. MCDougall EM, Soble JJ, Wolf J S J r, et al. Comparison of three-dimensional and two-dimensional laparoscopic video systems. J Endourol. 1996;10:371-74.

17. Mueller MD, Camartin C, Dreher E, et al. Three-dimensional laparoscopy. Gadget or progress? A randomized trial on the efficacy of three-dimensional laparoscopy. Surg Endosc. 1999;13:469-72.

18. Peitgen K, Walz MV, Walz MV, et al. A prospective randomized experimental evaluation of three-dimensional imaging in laparoscopy. Gastrointest Endosc. 1996;44: 262-7.

19. Perkins $N$, Starkes J L, Lee TD, et al. Learning to use minimal access surgical instruments and two-dimensional remote visual feedback: how difficult is the task for novices? Adv Health Sci Educ. 2002;7:117-31.

20. Schauer PR, Ikramuddin S, Luketich J D. Minilaparoscopy. Semin Laparosc Surg. 1999;6:21-31.

21. Scott DJ, J ones DB. Virtual reality training and teaching tools. In: Soper NJ , Swanstrom LL, Eubanks WS (eds). Mastery of Endoscopic and Laparoscopic Surgery. Lippincott Williams and Wilkins, Philadelphia. 2005;2:146-60.

22. Sun CC, Chiu AW, Chen KK, et al. Assessment of a three dimensional operating system with skill tests in a pelvic trainer. Urol Int. 2000;64:154-8.

23. Taffinder N, Smith SG, H uber J, et al. The effect of a secondgeneration $3 \mathrm{D}$ endoscope on the laparoscopic precision of novices and experienced surgeons. Surg Endosc. 1999;13:1087-92.

24. Thomsen MN, Lang RD. An experimental comparison of three-dimensional and two-dimensional endoscopic systems in a model. Arthroscopy. 2004;20:419-23.

25. Wickens $C D, H$ ollands]. Engineering psychology and human performance. Prentice Hall, Upper Saddle River. NJ $2000 ; 1164$. 